\title{
Analysis of Laminated Shells by Murakami's Zig-Zag Theory and Radial Basis Functions Collocation
}

\author{
D. A. Maturi, ${ }^{1}$ A. J. M. Ferreira, ${ }^{1,2}$ A. M. Zenkour, ${ }^{1,3}$ and D. S. Mashat ${ }^{1}$ \\ ${ }^{1}$ Department of Mathematics, Faculty of Science, King Abdulaziz University, P.O. Box 80203, Jeddah 21589, Saudi Arabia \\ ${ }^{2}$ Faculdade de Engenharia da Universidade do Porto, Porto, Portugal \\ ${ }^{3}$ Department of Mathematics, Faculty of Science, Kafrelsheikh University, Kafr El-Sheikh 33516, Egypt
}

Correspondence should be addressed to A. J. M. Ferreira; ferreira@fe.up.pt

Received 10 July 2013; Accepted 15 September 2013

Academic Editor: Song Cen

Copyright (C) 2013 D. A. Maturi et al. This is an open access article distributed under the Creative Commons Attribution License, which permits unrestricted use, distribution, and reproduction in any medium, provided the original work is properly cited.

\begin{abstract}
The static and free vibration analysis of laminated shells is performed by radial basis functions collocation, according to Murakami's zig-zag (ZZ) function (MZZF) theory . The MZZF theory accounts for through-the-thickness deformation, by considering a ZZ evolution of the transverse displacement with the thickness coordinate. The equations of motion and the boundary conditions are obtained by Carrera's Unified Formulation and further interpolated by collocation with radial basis functions.
\end{abstract}

\section{Introduction}

The efficient load-carrying capabilities of shell structures make them very useful in a variety of engineering applications. The continuous development of new structural materials leads to the ever increasingly complex structural designs that require careful analysis. Although analytical techniques are very important, the use of numerical methods to solve shell mathematical models of complex structures has become an essential ingredient in the design process.

The most common mathematical models used to describe shell structures may be classified into two classes according to different physical assumptions: the Koiter model [1], based on the Kirchhoff hypothesis, and the Naghdi model [2], based on the Reissner-Mindlin assumptions that take into account the transverse shear deformation. But these theories are not adequate to describe the so-called zig-zag (ZZ) effect in sandwich structures or layered composites, due to the discontinuity of mechanical properties between faces and core at the interfaces; see Figure 1 (to trace accurate responses of sandwich structures, see the books by Zenkert [3] and Vinson [4]).

The ZZ effect can be captured by the layerwise theories which typically assume independent degrees of freedom per layer. Unfortunately the computation can be prohibitive. The layerwise theories are reviewed in Burton and Noor
[5], Noor et al. [6], Altenbach [7], Librescu and Hause [8], Vinson [9], and Demasi [10]. In order to overcome the computational cost of the layerwise theories, Murakami [11] proposed a zig-zag function (ZZF) that is able to reproduce the slope discontinuity. Equivalent Single Layer models with only displacement unknowns can be developed on the basis of ZZF. A review of the application of ZZF in plates and shells was presented by Carrera [12-16] and some relevant papers on the analysis of sandwich structures were presented in [1719].

The most common numerical procedure for the analysis of the shells is the finite element method [20-24]. It is known that the phenomenon of numerical locking may arise from hidden constrains that are not well represented in the finite element approximation and, in the scientific literature, it is possible to find many methods to overcome this problem [25-30]. The present paper, that performs the bending and free vibration analysis of laminated shells by collocation with radial basis functions, avoids the locking phenomenon. A radial basis function, $\phi\left(\left\|x-x_{j}\right\|\right)$, is a spline that depends on the Euclidian distance between distinct data centers $x_{j, j}=1,2, \ldots, N \in \mathbb{R}^{n}$, also called nodal or collocation points. We use the so-called unsymmetrical Kansa method that was introduced by Kansa [31]. The use of radial basis function for the analysis of structures and materials has been previously studied by numerous authors [32-46]. The 


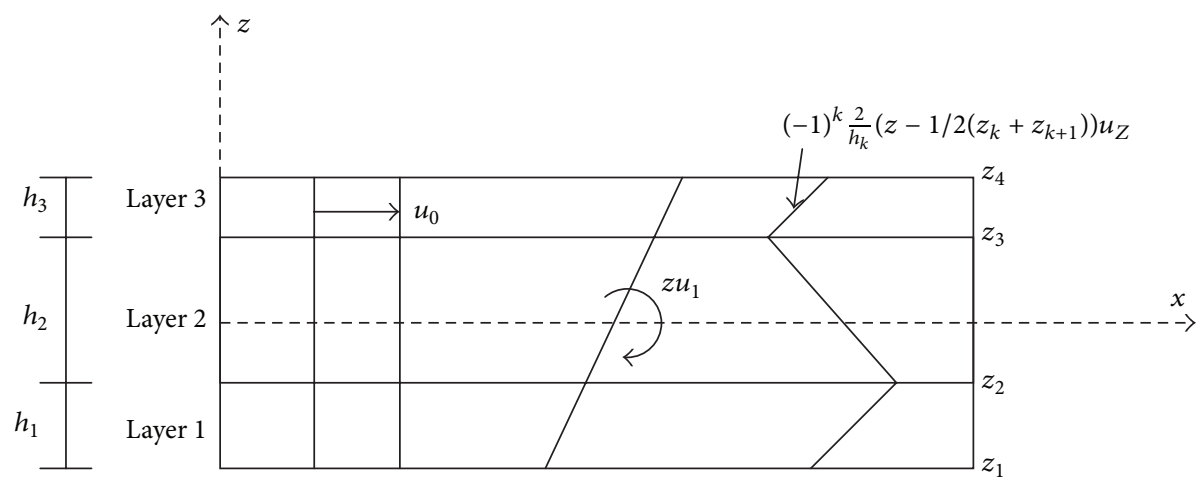

FIGURE 1: Scheme of the zig-zag assumptions for a three-layered laminate.

authors have recently applied the RBF collocation to the static deformations of composite beams and plates [47-49]. One of the authors has already combined Reddy's theory with radial basis functions in [50]. In fact, Reddy's theory is quite efficient for laminated (monolithic) composite plates or shells but not as efficient (or adequate) for sandwich structures because of the very high difference on material properties from the skins and the core. Reddy's theory does not allow the thickness stretching, but our formulation is more general and allows any expansion in the thickness direction. This is where the present paper shows more interest.

In this paper for the first time how the Unified Formulation can be combined with radial basis functions to the analysis of thin and thick laminated shells, using Murakami's zig-zag function, allowing for through-the-thickness deformations, is investigated. The quality of the present method in predicting static deformations and free vibrations of thin and thick laminated shells is compared and discussed with other methods in some numerical examples.

\section{Applying the Unified Formulation to MZZF}

The Unified Formulation (UF) proposed by Carrera $[13,51-$ 53], also known as CUF, is a powerful framework for the analysis of beams, plates, and shells. This formulation has been applied in several finite element analyses, either by using the Principle of Virtual Displacement, or by using Reissner's mixed variational theorem. The stiffness matrix components, the external force terms, or the inertia terms can be obtained directly with this UF, irrespective of the shear deformation theory being considered.

In this section Carrera's Unified Formulation is briefly reviewed. How to obtain the fundamental nuclei, which allows the derivation of the equations of motion and boundary conditions, in weak form for the finite element analysis and in strong form for the present RBF collocation, is shown.

2.1. The $M Z Z F$ Theory. Let us consider a sandwich plate (translation to shells becomes evident later in the paper) composed of three perfectly bonded layers, with $z$ being the thickness coordinate of the whole plate while $z_{k}$ is the layer thickness coordinate; see Figure 1. $a$ and $h$ are length and thickness of the square laminated plate, respectively. The adimensional layer coordinate $\zeta_{k}=\left(2 z_{k}\right) / h_{k}$ is further introduced ( $h_{k}$ is the thickness of the $k$ th layer). Murakami's zig-zag function $Z(z)$ was defined according to the following formula [11]:

$$
Z(z)=(-1)^{k} \zeta_{z}
$$

$Z(z)$ has the following properties.

(1) It is a piecewise linear function of layer coordinates $z_{k}$.

(2) $Z(z)$ has unit amplitude for the whole layers.

(3) The slope $Z^{\prime}(z)=d Z / d z$ assumes opposite sign between two adjacent layers. Its amplitude is layer thickness independent.

A possible FSDT theory has been investigated by Carrera [14] and Demasi [15], ignoring the through-the-thickness deformations:

$$
\begin{gathered}
u=u_{0}+z u_{1}+(-1)^{k} \frac{2}{h_{k}}\left(z-\frac{1}{2}\left(z_{k}+z_{k+1}\right)\right) u_{Z}, \\
v=v_{0}+z v_{1}+(-1)^{k} \frac{2}{h_{k}}\left(z-\frac{1}{2}\left(z_{k}+z_{k+1}\right)\right) v_{Z}, \\
w=w_{0} .
\end{gathered}
$$

A refinement of FSDT by inclusion of $\mathrm{ZZ}$ effects and transverse normal strains was introduced in Murakami's original ZZF, defined by the following displacement field:

$$
\begin{gathered}
u=u_{0}+z u_{1}+(-1)^{k} \frac{2}{h_{k}}\left(z-\frac{1}{2}\left(z_{k}+z_{k+1}\right)\right) u_{Z}, \\
v=v_{0}+z v_{1}+(-1)^{k} \frac{2}{h_{k}}\left(z-\frac{1}{2}\left(z_{k}+z_{k+1}\right)\right) v_{Z}, \\
w=w_{0}+z w_{1}+(-1)^{k} \frac{2}{h_{k}}\left(z-\frac{1}{2}\left(z_{k}+z_{k+1}\right)\right) w_{Z},
\end{gathered}
$$

where $z_{k}$ and $z_{k+1}$ are the bottom and top $z$-coordinates at each layer. The additional degrees of freedom $u_{Z}$ and $v_{Z}$ have a meaning of displacement, and their amplitude is layer independent. 
2.2. Governing Equations and Boundary Conditions in the Framework of Unified Formulation. Shells are bidimensional structures in which one dimension (in general the thickness in $z$ direction) is negligible with respect to the other two inplane dimensions. Geometry and the reference system are indicated in Figure 3. The square of an infinitesimal linear segment in the layer and the associated infinitesimal area and volume are given by

$$
\begin{gathered}
d s_{k}^{2}=H_{\alpha}^{k^{2}} d \alpha^{2}+H_{\beta}^{k^{2}} d \beta^{2}+H_{z}^{k^{2}} d z^{2}, \\
d \Omega_{k}=H_{\alpha}^{k} H_{\beta}^{k} d \alpha d \beta, \\
d V=H_{\alpha}^{k} H_{\beta}^{k} H_{z}^{k} d \alpha d \beta d z,
\end{gathered}
$$

where the metric coefficients are

$$
\begin{gathered}
H_{\alpha}^{k}=A^{k}\left(1+\frac{z}{R_{\alpha}^{k}}\right), \\
H_{\beta}^{k}=B^{k}\left(1+\frac{z}{R_{\beta}^{k}}\right), \quad H_{z}^{k}=1 .
\end{gathered}
$$

$k$ denotes the $k$-layer of the multilayered shell; $R_{\alpha}^{k}$ and $R_{\beta}^{k}$ are the principal radii of curvature along the coordinates $\alpha$ and $\beta$, respectively. $A^{k}$ and $B^{k}$ are the coefficients of the first fundamental form of $\Omega_{k}$ ( $\Gamma_{k}$ is the $\Omega_{k}$ boundary). In this work, the attention has been restricted to shells with constant radii of curvature (cylindrical, spherical, and toroidal geometries) for which $A^{k}=B^{k}=1$.

Although one can use the UF for one-layer, isotropic shell, a multilayered shell with $N_{l}$ layers is considered. The Principle of Virtual Displacement (PVD) for the pure-mechanical case reads

$$
\sum_{k=1}^{N_{l}} \int_{\Omega_{k}} \int_{A_{k}}\left\{\delta \boldsymbol{\epsilon}_{p G}^{k T} \boldsymbol{\sigma}_{p C}^{k}+\delta \boldsymbol{\epsilon}_{n G}^{k T} \boldsymbol{\sigma}_{n C}^{k}\right\} \times d \Omega_{k} d z=\sum_{k=1}^{N_{l}} \delta L_{e}^{k},
$$

where $\Omega_{k}$ and $A_{k}$ are the integration domains in plane $(\alpha, \beta)$ and $z$ direction, respectively. Here, $k$ indicates the layer and $T$ the transpose of a vector, and $\delta L_{e}^{k}$ is the external work for the $k$ th layer. $G$ means geometrical relations and $C$ constitutive equations.

The steps to obtain the governing equations are

(i) substitution of the geometrical relations (subscript $G)$,

(ii) substitution of the appropriate constitutive equations (subscript $C$ ),

(iii) introduction of the Unified Formulation.

Stresses and strains are separated into in-plane and normal components, denoted, respectively, by the subscripts $p$ and $n$. The mechanical strains in the $k$ th layer can be related to the displacement field $\mathbf{u}^{k}=\left\{u_{\alpha}^{k}, u_{\beta}^{k}, u_{z}^{k}\right\}$ via the geometrical relations:

$$
\begin{gathered}
\epsilon_{p G}^{k}=\left[\epsilon_{\alpha \alpha}^{k}, \epsilon_{\beta \beta}^{k}, \epsilon_{\alpha \beta}^{k}\right]^{T}=\left(\mathbf{D}_{p}^{k}+\mathbf{A}_{p}^{k}\right) \mathbf{u}^{k}, \\
\epsilon_{n G}^{k}=\left[\epsilon_{\alpha z}^{k}, \epsilon_{\beta z}^{k}, \epsilon_{z z}^{k}\right]^{T}=\left(\mathbf{D}_{n \Omega}^{k}+\mathbf{D}_{n z}^{k}-\mathbf{A}_{n}^{k}\right) \mathbf{u}^{k} .
\end{gathered}
$$

The explicit form of the introduced arrays is as follows

$$
\begin{gathered}
\mathbf{D}_{p}^{k}=\left[\begin{array}{ccc}
\frac{\partial_{\alpha}}{H_{\alpha}^{k}} & 0 & 0 \\
0 & \frac{\partial_{\beta}}{H_{\beta}^{k}} & 0 \\
\frac{\partial_{\beta}}{H_{\beta}^{k}} & \frac{\partial_{\alpha}}{H_{\alpha}^{k}} & 0
\end{array}\right], \quad \mathbf{D}_{n \Omega}^{k}=\left[\begin{array}{ccc}
0 & 0 & \frac{\partial_{\alpha}}{H_{\alpha}^{k}} \\
0 & 0 & \frac{\partial_{\beta}}{H_{\beta}^{k}} \\
0 & 0 & 0
\end{array}\right], \\
\left.\mathbf{A}_{p}^{k}=\left[\begin{array}{lll}
0 & 0 & \frac{1}{H_{\alpha}^{k} R_{\alpha}^{k}} \\
0 & 0 & \frac{1}{H_{\beta}^{k} R_{\beta}^{k}} \\
0 & 0 & 0
\end{array}\right], \quad \begin{array}{ccc}
\partial_{z} & 0 & 0 \\
0 & \partial_{z} & 0 \\
0 & 0 & \partial_{z}
\end{array}\right], \\
\mathbf{A}_{n}^{k}=\left[\begin{array}{ccc}
\frac{1}{H_{\alpha}^{k} R_{\alpha}^{k}} & 0 & 0 \\
0 & \frac{1}{H_{\beta}^{k} R_{\beta}^{k}} & 0 \\
0 & 0 & 0
\end{array}\right] .
\end{gathered}
$$

The $3 \mathrm{D}$ constitutive equations are given as

$$
\begin{gathered}
\boldsymbol{\sigma}_{p C}^{k}=\mathbf{C}_{p p}^{k} \boldsymbol{\epsilon}_{p G}^{k}+\mathbf{C}_{p n}^{k} \boldsymbol{\epsilon}_{n G}^{k}, \\
\boldsymbol{\sigma}_{n C}^{k}=\mathbf{C}_{n p}^{k} \boldsymbol{\epsilon}_{p G}^{k}+\mathbf{C}_{n n}^{k} \boldsymbol{\epsilon}_{n G}^{k}
\end{gathered}
$$

with

$$
\begin{gathered}
\mathbf{C}_{p p}^{k}=\left[\begin{array}{lll}
C_{11}^{k} & C_{12}^{k} & C_{16}^{k} \\
C_{12}^{k} & C_{22}^{k} & C_{26}^{k} \\
C_{16}^{k} & C_{26}^{k} & C_{66}^{k}
\end{array}\right], \quad \mathbf{C}_{p n}^{k}=\left[\begin{array}{lll}
0 & 0 & C_{13}^{k} \\
0 & 0 & C_{23}^{k} \\
0 & 0 & C_{36}^{k}
\end{array}\right], \\
\mathbf{C}_{n p}^{k}=\left[\begin{array}{ccc}
0 & 0 & 0 \\
0 & 0 & 0 \\
C_{13}^{k} & C_{23}^{k} & C_{36}^{k}
\end{array}\right], \quad \mathbf{C}_{n n}^{k}=\left[\begin{array}{ccc}
C_{55}^{k} & C_{45}^{k} & 0 \\
C_{45}^{k} & C_{44}^{k} & 0 \\
0 & 0 & C_{33}^{k}
\end{array}\right] .
\end{gathered}
$$

According to the Unified Formulation by Carrera, the three displacement components $u_{\alpha}, u_{\beta}$, and $u_{z}$ and their relative variations can be modelled as

$$
\begin{array}{r}
\left(u_{\alpha}, u_{\beta}, u_{z}\right)=F_{\tau}\left(u_{\alpha \tau}, u_{\beta \tau}, u_{z \tau}\right), \\
\left(\delta u_{\alpha}, \delta u_{\beta}, \delta u_{z}\right)=F_{s}\left(\delta u_{\alpha s}, \delta u_{\beta s}, \delta u_{z s}\right)
\end{array}
$$

with Taylor expansions from the first up to the 4th order: $F_{0}=$ $z^{0}=1, F_{1}=z^{1}=z, \ldots, F_{N}=z^{N}, \ldots$, and $F_{4}=z^{4}$ if an Equivalent Single Layer (ESL) approach is used.

Resorting to the displacement field in (3), we choose vectors $F_{t}=\left[1 z(-1)^{k}\left(2 / h_{k}\right)\left(z-(1 / 2)\left(z_{k}+z_{k+1}\right)\right)\right]$ for displacement $u, v$, and $w$. We then obtain all terms of the equations of motion by integrating through-the-thickness direction.

It is interesting to note that, under this combination of the Unified Formulation and RBF collocation, the collocation code depends only on the choice of $F_{t}$, in order to solve this type of problems. We designed a MATLAB code that just by changing $F_{t}$ can analyse static deformations, free vibrations, 


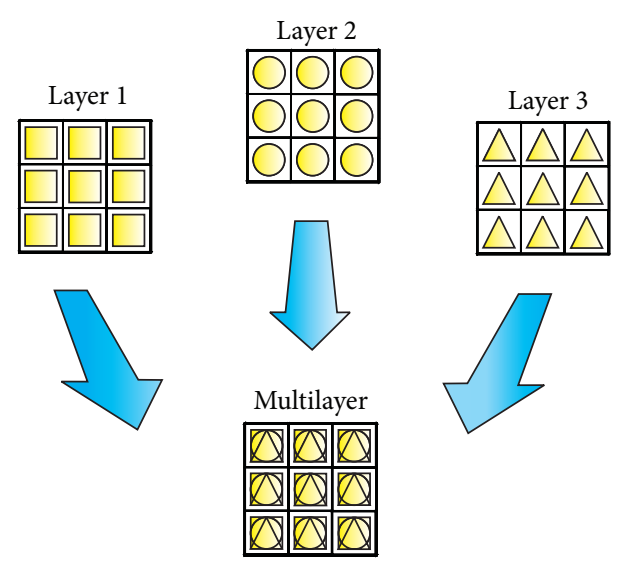

Figure 2: Assembling procedure for ESL approach.

and buckling loads for any type of $C^{\circ}$ shear deformation theory. An obvious advantage of the present methodology is that the tedious derivation of the equations of motion and boundary conditions for a particular shear deformation theory is no longer an issue, as this MATLAB code does all that work for us.

In Figure 2 the assembling procedures are shown on layer $k$ for ESL approach.

Substituting the geometrical relations, the constitutive equations, and the Unified Formulation into the variational statement PVD, for the $k$ th layer, one has

$$
\begin{aligned}
\sum_{k=1}^{N_{l}}\left\{\int_{\Omega_{k}} \int_{A_{k}}\{\right. & \left(\left(\mathbf{D}_{p}+\mathbf{A}_{p}\right) \delta \mathbf{u}^{k}\right)^{T} \\
& \times\left(\mathbf{C}_{p p}^{k}\left(\mathbf{D}_{p}+\mathbf{A}_{p}\right) \mathbf{u}^{k}\right. \\
& \left.\quad+\mathbf{C}_{p n}^{k}\left(\mathbf{D}_{n \Omega}+\mathbf{D}_{n z}-\mathbf{A}_{n}\right) \mathbf{u}^{k}\right) \\
+ & \left(\left(\mathbf{D}_{n \Omega}+\mathbf{D}_{n z}-\mathbf{A}_{n}\right) \delta \mathbf{u}^{k}\right)^{T} \\
& \times\left(\mathbf{C}_{n p}^{k}\left(\mathbf{D}_{p}+\mathbf{A}_{p}\right) \mathbf{u}^{k}\right. \\
& \left.\left.\left.+\mathbf{C}_{n n}^{k}\left(\mathbf{D}_{n \Omega}+\mathbf{D}_{n z}-\mathbf{A}_{n}\right) \mathbf{u}^{k}\right)\right\} d \Omega_{k} d z_{k}\right\} \\
= & \sum_{k=1}^{N_{l}} \delta L_{e}^{k} .
\end{aligned}
$$

At this point, the formula of integration by parts is applied

$$
\begin{aligned}
\int_{\Omega_{k}}\left(\left(\mathbf{D}_{\Omega}\right) \delta \mathbf{a}^{k}\right)^{T} \mathbf{a}^{k} d \Omega_{k}= & -\int_{\Omega_{k}} \delta \mathbf{a}^{k^{T}}\left(\left(\mathbf{D}_{\Omega}^{T}\right) \mathbf{a}^{k}\right) d \Omega_{k} \\
& +\int_{\Gamma_{k}} \delta \mathbf{a}^{k^{T}}\left(\left(\mathbf{I}_{\Omega}\right) \mathbf{a}^{k}\right) d \Gamma_{k},
\end{aligned}
$$

where $\mathbf{I}_{\Omega}$ matrix is obtained applying the gradient theorem

$$
\int_{\Omega} \frac{\partial \psi}{\partial x_{i}} d v=\oint_{\Gamma} n_{i} \psi d s
$$

with $n_{i}$ being the components of the normal $\widehat{n}$ to the boundary along the direction $i$. After integration by parts and the substitution of CUF, the governing equations and boundary conditions for the shell in the mechanical case are obtained:

$$
\begin{aligned}
& \sum_{k=1}^{N_{l}}\left\{\int _ { \Omega _ { k } } \int _ { A _ { k } } \left\{\delta \mathbf { u } _ { s } ^ { k T } \left[\left(-\mathbf{D}_{p}+\mathbf{A}_{p}\right)^{T}\right.\right.\right. \\
& \times F_{s}\left(\mathbf{C}_{p p}^{k}\left(\mathbf{D}_{p}+\mathbf{A}_{p}\right) F_{\tau} \mathbf{u}_{\tau}^{k}\right. \\
& \left.\left.+\mathbf{C}_{p n}^{k}\left(\mathbf{D}_{n \Omega}+\mathbf{D}_{n z}-\mathbf{A}_{n}\right) F_{\tau} \mathbf{u}_{\tau}^{k}\right)\right] \\
& +\delta \mathbf{u}_{s}^{k T}\left[\left(-\mathbf{D}_{n \Omega}+\mathbf{D}_{n z}-\mathbf{A}_{n}\right)^{T}\right. \\
& \times F_{s}\left(\mathbf{C}_{n p}^{k}\left(\mathbf{D}_{p}+\mathbf{A}_{p}\right) F_{\tau} \mathbf{u}_{\tau}^{k}\right. \\
& +\mathbf{C}_{n n}^{k}\left(\mathbf{D}_{n \Omega}+\mathbf{D}_{n z}\right. \\
& \left.\left.\left.\left.\left.-\mathbf{A}_{n}\right) F_{\tau} \mathbf{u}_{\tau}^{k}\right)\right]\right\} d \Omega_{k} d z_{k}\right\} \\
& +\sum_{k=1}^{N_{l}}\left\{\int _ { \Gamma _ { k } } \int _ { A _ { k } } \left\{\delta \mathbf { u } _ { s } ^ { k T } \left[\mathbf { I } _ { p } ^ { T } F _ { s } \left(\mathbf{C}_{p p}^{k}\left(\mathbf{D}_{p}+\mathbf{A}_{p}\right) F_{\tau} \mathbf{u}_{\tau}^{k}\right.\right.\right.\right. \\
& +\mathbf{C}_{p n}^{k}\left(\mathbf{D}_{n \Omega}+\mathbf{D}_{n z}\right. \\
& \left.\left.\left.-\mathbf{A}_{n}\right) F_{\tau} \mathbf{u}_{\tau}^{k}\right)\right] \\
& +\delta \mathbf{u}_{s}^{k T}\left[\mathbf { I } _ { n p } ^ { T } F _ { s } \left(\mathbf{C}_{n p}^{k}\left(\mathbf{D}_{p}-\mathbf{A}_{p}\right) F_{\tau} \mathbf{u}_{\tau}^{k}\right.\right. \\
& +\mathbf{C}_{n n}^{k}\left(\mathbf{D}_{n \Omega}+\mathbf{D}_{n z}-\mathbf{A}_{n}\right) \\
& \left.\left.\left.\left.\times F_{\tau} \mathbf{u}_{\tau}^{k}\right)\right]\right\} d \Gamma_{k} d z_{k}\right\} \\
& =\sum_{k=1}^{N_{l}}\left\{\int_{\Omega_{k}} \delta \mathbf{u}_{s}^{k T} F_{s} \mathbf{p}_{u}^{k}\right\}
\end{aligned}
$$

where $\mathbf{I}_{p}^{k}$ and $\mathbf{I}_{n p}^{k}$ depend on the boundary geometry:

$$
\mathbf{I}_{p}=\left[\begin{array}{ccc}
\frac{n_{\alpha}}{H_{\alpha}} & 0 & 0 \\
0 & \frac{n_{\beta}}{H_{\beta}} & 0 \\
\frac{n_{\beta}}{H_{\beta}} & \frac{n_{\alpha}}{H_{\alpha}} & 0
\end{array}\right], \quad \mathbf{I}_{n p}=\left[\begin{array}{ccc}
0 & 0 & \frac{n_{\alpha}}{H_{\alpha}} \\
0 & 0 & \frac{n_{\beta}}{H_{\beta}} \\
0 & 0 & 0
\end{array}\right] .
$$

The normal to the boundary of domain $\Omega$ is

$$
\widehat{\mathbf{n}}=\left[\begin{array}{l}
n_{\alpha} \\
n_{\beta}
\end{array}\right]=\left[\begin{array}{c}
\cos \left(\varphi_{\alpha}\right) \\
\cos \left(\varphi_{\beta}\right)
\end{array}\right],
$$

where $\varphi_{\alpha}$ and $\varphi_{\beta}$ are the angles between the normal $\widehat{n}$ and the directions $\alpha$ and $\beta$, respectively.

The governing equations for a multilayered shell subjected to mechanical loadings are

$$
\delta \mathbf{u}_{s}^{k^{T}}: \mathbf{K}_{u u}^{k \tau s} \mathbf{u}_{\tau}^{k}=\mathbf{P}_{u \tau}^{k},
$$




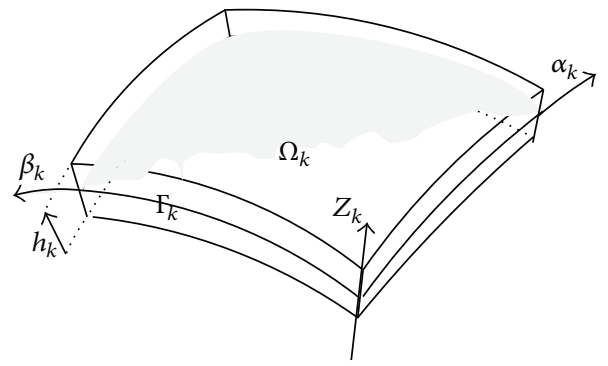

(a)

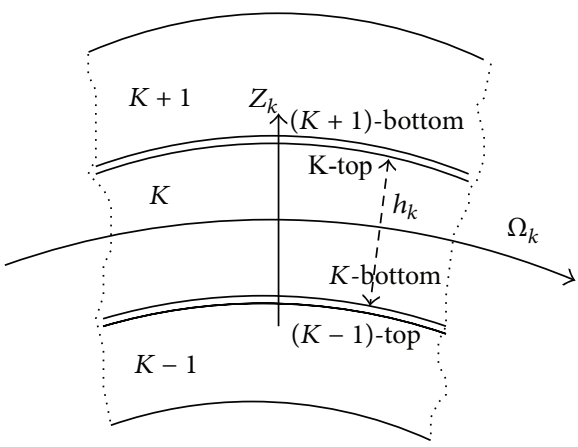

(b)

FIGURE 3: Geometry and notations for a multilayered shell (doubly curved).

where the fundamental nucleus $\mathbf{K}_{u u}^{k \tau s}$ is obtained as

$$
\begin{aligned}
\mathbf{K}_{u u}^{k \tau s}=\int_{A_{k}}[ & {\left[-\mathbf{D}_{p}+\mathbf{A}_{p}\right]^{T} \mathbf{C}_{p p}^{k}\left[\mathbf{D}_{p}+\mathbf{A}_{p}\right] } \\
& +\left[-\mathbf{D}_{p}+\mathbf{A}_{p}\right]^{T} \mathbf{C}_{p n}^{k}\left[\mathbf{D}_{n \Omega}+\mathbf{D}_{n z}-\mathbf{A}_{n}\right] \\
& +\left[-\mathbf{D}_{n \Omega}+\mathbf{D}_{n z}-\mathbf{A}_{n}\right]^{T} \mathbf{C}_{n p}^{k}\left[\mathbf{D}_{p}+\mathbf{A}_{p}\right] \\
& +\left[-\mathbf{D}_{n \Omega}+\mathbf{D}_{n z}-\mathbf{A}_{n}\right]^{T} \\
& \left.\times \mathbf{C}_{n n}^{k}\left[\mathbf{D}_{n \Omega}+\mathbf{D}_{n z}-\mathbf{A}_{n}\right]\right] F_{\tau} F_{s} H_{\alpha}^{k} H_{\beta}^{k} d z .
\end{aligned}
$$

And the corresponding Neumann-type boundary conditions on $\Gamma_{k}$ are

$$
\Pi_{d}^{k \tau s} \mathbf{u}_{\tau}^{k}=\Pi_{d}^{k \tau s} \overline{\mathbf{u}}_{\tau}^{k} \text {, }
$$

where

$$
\begin{aligned}
& \boldsymbol{\Pi}_{d}^{k \tau s}=\int_{A_{k}}\left[\mathbf{I}_{p}^{T} \mathbf{C}_{p p}^{k}\left[\mathbf{D}_{p}+\mathbf{A}_{p}^{\tau}\right]+\mathbf{I}_{p}^{T} \mathbf{C}_{p n}^{k}\left[\mathbf{D}_{n \Omega}+\mathbf{D}_{n z}-\mathbf{A}_{n}^{\tau}\right]\right. \\
& +\mathbf{I}_{n p}^{T} \mathbf{C}_{n p}^{k}\left[\mathbf{D}_{p}+\mathbf{A}_{p}^{\tau}\right]+\mathbf{I}_{n p}^{T} \mathbf{C}_{n n}^{k} \\
& \left.\times\left[\mathbf{D}_{n \Omega}+\mathbf{D}_{n z}-\mathbf{A}_{n}^{\tau}\right]\right] F_{\tau} F_{s} H_{\alpha}^{k} H_{\beta}^{k} d z \text {. }
\end{aligned}
$$

And $\mathbf{P}_{u \tau}^{k}$ are variationally consistent loads with applied pressure.

2.3. Fundamental Nuclei. The following integrals are introduced to perform the explicit form of fundamental nuclei:

$$
\begin{aligned}
& \left(J^{k \tau s}, J_{\alpha}^{k \tau s}, J_{\beta}^{k \tau s}, J_{\alpha / \beta}^{k \tau s}, J_{\beta / \alpha}^{k \tau s}, J_{\alpha \beta}^{k \tau s}\right) \\
& =\int_{A_{k}} F_{\tau} F_{s}\left(1, H_{\alpha}, H_{\beta}, \frac{H_{\alpha}}{H_{\beta}}, \frac{H_{\beta}}{H_{\alpha}}, H_{\alpha} H_{\beta}\right) d z, \\
& \left(J^{k \tau_{z} s}, J_{\alpha}^{k \tau_{z} s}, J_{\beta}^{k \tau_{z} s}, J_{\alpha / \beta}^{k \tau_{z} s}, J_{\beta / \alpha}^{k \tau_{z} s}, J_{\alpha \beta}^{k \tau_{z} s}\right) \\
& =\int_{A_{k}} \frac{\partial F_{\tau}}{\partial z} F_{s}\left(1, H_{\alpha}, H_{\beta}, \frac{H_{\alpha}}{H_{\beta}}, \frac{H_{\beta}}{H_{\alpha}}, H_{\alpha} H_{\beta}\right) d z,
\end{aligned}
$$

$$
\begin{aligned}
& \left(J^{k \tau s_{z}}, J_{\alpha}^{k \tau s_{z}}, J_{\beta}^{k \tau s_{z}}, J_{\alpha / \beta}^{k \tau s_{z}}, J_{\beta / \alpha}^{k \tau s_{z}}, J_{\alpha \beta}^{k \tau s_{z}}\right) \\
& =\int_{A_{k}} F_{\tau} \frac{\partial F_{s}}{\partial z}\left(1, H_{\alpha}, H_{\beta}, \frac{H_{\alpha}}{H_{\beta}}, \frac{H_{\beta}}{H_{\alpha}}, H_{\alpha} H_{\beta}\right) d z, \\
& \left(J^{k \tau_{z} s_{z}}, J_{\alpha}^{k \tau_{z} s_{z}}, J_{\beta}^{k \tau_{z} s_{z}}, J_{\alpha / \beta}^{k \tau_{z} s_{z}}, J_{\beta / \alpha}^{k \tau_{z} s_{z}}, J_{\alpha \beta}^{k \tau_{z} s_{z}}\right) \\
& =\int_{A_{k}} \frac{\partial F_{\tau}}{\partial z} \frac{\partial F_{s}}{\partial z}\left(1, H_{\alpha}, H_{\beta}, \frac{H_{\alpha}}{H_{\beta}}, \frac{H_{\beta}}{H_{\alpha}}, H_{\alpha} H_{\beta}\right) d z .
\end{aligned}
$$

The fundamental nucleus $\mathbf{K}_{u u}^{k \tau s}$ is reported for doubly curved shells (radii of curvature in both $\alpha$ and $\beta$ directions; see Figure 3):

$$
\begin{aligned}
\left(\mathbf{K}_{u u}^{\tau s k}\right)_{11}= & -C_{11}^{k} J_{\beta / \alpha}^{k \tau s} \partial_{\alpha}^{s} \partial_{\alpha}^{\tau}-C_{16}^{k} J^{k \tau s} \partial_{\alpha}^{\tau} \partial_{\beta}^{s} \\
& -C_{16}^{k} J^{k \tau s} \partial_{\alpha}^{s} \partial_{\beta}^{\tau}-C_{66}^{k} J_{\alpha / \beta}^{k \tau s} \partial_{\beta}^{s} \partial_{\beta}^{\tau} \\
& +C_{55}^{k}\left(J_{\alpha \beta}^{k \tau_{z} s_{z}}-\frac{1}{R_{\alpha_{k}}} J_{\beta}^{k \tau_{z} s}\right. \\
& \left.-\frac{1}{R_{\alpha_{k}}} J_{\beta}^{k \tau s_{z}}+\frac{1}{R_{\alpha_{k}}^{2}} J_{\beta / \alpha}^{k \tau s}\right), \\
\left(\mathbf{K}_{u u}^{\tau s k}\right)_{12}= & -C_{12}^{k} J^{k \tau s} \partial_{\alpha}^{\tau} \partial_{\beta}^{s}-C_{16}^{k} J_{\beta / \alpha}^{k \tau s} \partial_{\alpha}^{s} \partial_{\alpha}^{\tau} \\
& -C_{26}^{k} J_{\alpha / \beta}^{k \tau s} \partial_{\beta}^{s} \partial_{\beta}^{\tau}-C_{66}^{k} J^{k \tau s} \partial_{\alpha}^{s} \partial_{\beta}^{\tau} \\
& +C_{45}^{k}\left(J_{\alpha \beta}^{k \tau_{z} s_{z}}-\frac{1}{R_{\beta_{k}}} J_{\alpha}^{k \tau_{z} s}\right. \\
& \left.-\frac{1}{R_{\alpha_{k}}} J_{\beta}^{k \tau s_{z}}+\frac{1}{R_{\alpha_{k}}} \frac{1}{R_{\beta_{k}}} J^{k \tau s}\right), \\
\left(\mathbf{K}_{u u}^{\tau s k}\right)_{13}= & -C_{11}^{k} \frac{1}{R_{\alpha_{k}}} J_{\beta / \alpha}^{k \tau s} \partial_{\alpha}^{\tau}-C_{12}^{k} \frac{1}{R_{\beta_{k}}} J^{k \tau s} \partial_{\alpha}^{\tau} \\
& -C_{13}^{k} J_{\beta}^{k \tau s_{z}} \partial_{\alpha}^{\tau}-C_{16}^{k} \frac{1}{R_{\alpha_{k}}} J^{k \tau s} \partial_{\beta}^{\tau}
\end{aligned}
$$




$$
\begin{aligned}
& -C_{26}^{k} \frac{1}{R_{\beta_{k}}} J_{\alpha / \beta}^{k \tau s} \partial_{\beta}^{\tau}-C_{36}^{k} J_{\alpha}^{k \tau s_{z}} \partial_{\beta}^{\tau} \\
& +C_{45}^{k}\left(J_{\alpha}^{k \tau_{z} s} \partial_{\beta}^{s}-\frac{1}{R_{\alpha_{k}}} J^{k \tau s} \partial_{\beta}^{s}\right) \\
& +C_{55}^{k}\left(J_{\beta}^{k \tau_{z} s} \partial_{\alpha}^{s}-\frac{1}{R_{\alpha_{k}}} J_{\beta / \alpha}^{k \tau s} \partial_{\alpha}^{s}\right), \\
& \left(\mathbf{K}_{u u}^{\tau s k}\right)_{21}=-C_{12}^{k} J^{k \tau s} \partial_{\alpha}^{s} \partial_{\beta}^{\tau}-C_{16}^{k} J_{\beta / \alpha}^{k \tau s} \partial_{\alpha}^{s} \partial_{\alpha}^{\tau} \\
& -C_{26}^{k} J_{\alpha / \beta}^{k \tau s} \partial_{\beta}^{s} \partial_{\beta}^{\tau}-C_{66}^{k} J^{k \tau s} \partial_{\alpha}^{\tau} \partial_{\beta}^{s} \\
& +C_{45}^{k}\left(J_{\alpha \beta}^{k \tau_{z} s_{z}}-\frac{1}{R_{\beta_{k}}} J_{\alpha}^{k \tau s_{z}}\right. \\
& \left.-\frac{1}{R_{\alpha_{k}}} J_{\beta}^{k \tau_{z} s}+\frac{1}{R_{\alpha_{k}}} \frac{1}{R_{\beta_{k}}} J^{k \tau s}\right), \\
& \left(\mathbf{K}_{u u}^{\tau s k}\right)_{22}=-C_{22}^{k} J_{\alpha / \beta}^{k \tau s} \partial_{\beta}^{s} \partial_{\beta}^{\tau}-C_{26}^{k} J^{k \tau s} \partial_{\alpha}^{s} \partial_{\beta}^{\tau} \\
& -C_{26}^{k} J^{k \tau s} \partial_{\alpha}^{\tau} \partial_{\beta}^{s}-C_{66}^{k} J_{\beta / \alpha}^{k \tau s} \partial_{\alpha}^{s} \partial_{\alpha}^{\tau} \\
& +C_{44}^{k}\left(J_{\alpha \beta}^{k \tau_{z} s_{z}}-\frac{1}{R_{\beta_{k}}} J_{\alpha}^{k \tau_{z} s}\right. \\
& \left.-\frac{1}{R_{\beta_{k}}} J_{\alpha}^{k \tau s_{z}}+\frac{1}{R_{\beta_{k}}^{2}} J_{\alpha / \beta}^{k \tau s}\right), \\
& \left(\mathbf{K}_{u u}^{\tau s k}\right)_{23}=-C_{12}^{k} \frac{1}{R_{\alpha_{k}}} J^{k \tau s} \partial_{\beta}^{\tau}-C_{22}^{k} \frac{1}{R_{\beta_{k}}} J_{\alpha / \beta}^{k \tau s} \partial_{\beta}^{\tau} \\
& -C_{23}^{k} J_{\alpha}^{k \tau s_{z}} \partial_{\beta}^{\tau}-C_{16}^{k} \frac{1}{R_{\alpha_{k}}} J_{\beta / \alpha}^{k \tau s} \partial_{\alpha}^{\tau} \\
& -C_{26}^{k} \frac{1}{R_{\beta_{k}}} J^{k \tau s} \partial_{\alpha}^{\tau}-C_{36}^{k} J_{\beta}^{k \tau s_{z}} \partial_{\alpha}^{\tau} \\
& +C_{45}^{k}\left(J_{\beta}^{k \tau_{z} s} \partial_{\alpha}^{s}-\frac{1}{R_{\beta_{k}}} J^{k \tau s} \partial_{\alpha}^{s}\right) \\
& +C_{44}^{k}\left(J_{\alpha}^{k \tau_{z} s} \partial_{\beta}^{s}-\frac{1}{R_{\beta_{k}}} J_{\alpha / \beta}^{k \tau s} \partial_{\beta}^{s}\right), \\
& \left(\mathbf{K}_{u u}^{\tau s k}\right)_{31}=C_{11}^{k} \frac{1}{R_{\alpha_{k}}} J_{\beta / \alpha}^{k \tau s} \partial_{\alpha}^{s}+C_{12}^{k} \frac{1}{R_{\beta_{k}}} J^{k \tau s} \partial_{\alpha}^{s} \\
& +C_{13}^{k} J_{\beta}^{k \tau_{z} s} \partial_{\alpha}^{s}+C_{16}^{k} \frac{1}{R_{\alpha_{k}}} J^{k \tau s} \partial_{\beta}^{s} \\
& +C_{26}^{k} \frac{1}{R_{\beta_{k}}} J_{\alpha / \beta}^{k \tau s} \partial_{\beta}^{s}+C_{36}^{k} J_{\alpha}^{k \tau_{z} s} \partial_{\beta}^{s} \\
& -C_{45}^{k}\left(J_{\alpha}^{k \tau s_{z}} \partial_{\beta}^{\tau}-\frac{1}{R_{\alpha_{k}}} J^{k \tau s} \partial_{\beta}^{\tau}\right) \\
& -C_{55}^{k}\left(J_{\beta}^{k \tau s_{z}} \partial_{\alpha}^{\tau}-\frac{1}{R_{\alpha_{k}}} J_{\beta / \alpha}^{k \tau s} \partial_{\alpha}^{\tau}\right)
\end{aligned}
$$

$$
\begin{aligned}
& \left(\mathbf{K}_{u u}^{\tau s k}\right)_{32}=C_{12}^{k} \frac{1}{R_{\alpha_{k}}} J^{k \tau s} \partial_{\beta}^{s}+C_{22}^{k} \frac{1}{R_{\beta_{k}}} J_{\alpha / \beta}^{k \tau s} \partial_{\beta}^{s} \\
& +C_{23}^{k} J_{\alpha}^{k \tau_{z} s} \partial_{\beta}^{s}+C_{16}^{k} \frac{1}{R_{\alpha_{k}}} J_{\beta / \alpha}^{k \tau s} \partial_{\alpha}^{s} \\
& +C_{26}^{k} \frac{1}{R_{\beta_{k}}} J^{k \tau s} \partial_{\alpha}^{s}+C_{36}^{k} J_{\beta}^{k \tau_{z} s} \partial_{\alpha}^{s} \\
& -C_{45}^{k}\left(J_{\beta}^{k \tau s_{z}} \partial_{\alpha}^{\tau}-\frac{1}{R_{\beta_{k}}} J^{k \tau s} \partial_{\alpha}^{\tau}\right) \\
& -C_{44}^{k}\left(J_{\alpha}^{k \tau s_{z}} \partial_{\beta}^{\tau}-\frac{1}{R_{\beta_{k}}} J_{\alpha / \beta}^{k \tau s} \partial_{\beta}^{\tau}\right), \\
& \left(\mathbf{K}_{u u}^{\tau s k}\right)_{33}=C_{11}^{k} \frac{1}{R_{\alpha_{k}}^{2}} J_{\beta / \alpha}^{k \tau s}+C_{22}^{k} \frac{1}{R_{\beta_{k}}^{2}} J_{\alpha / \beta}^{k \tau s} \\
& +C_{33}^{k} J_{\alpha \beta}^{k \tau_{z} s_{z}}+2 C_{12}^{k} \frac{1}{R_{\alpha_{k}}} \frac{1}{R_{\beta_{k}}} J^{k \tau s} \\
& +C_{13}^{k} \frac{1}{R_{\alpha_{k}}}\left(J_{\beta}^{k \tau_{z} s}+J_{\beta}^{k \tau s_{z}}\right) \\
& +C_{23}^{k} \frac{1}{R_{\beta_{k}}}\left(J_{\alpha}^{k \tau_{z} s}+J_{\alpha}^{k \tau s_{z}}\right) \\
& -C_{44}^{k} J_{\alpha / \beta}^{k \tau s} \partial_{\beta}^{s} \partial_{\beta}^{\tau}-C_{55}^{k} J_{\beta / \alpha}^{k \tau s} \partial_{\alpha}^{s} \partial_{\alpha}^{\tau} \\
& -C_{45}^{k} J^{k \tau s} \partial_{\alpha}^{s} \partial_{\beta}^{\tau}-C_{45}^{k} J^{k \tau s} \partial_{\alpha}^{\tau} \partial_{\beta}^{s} .
\end{aligned}
$$

The application of boundary conditions makes use of the fundamental nucleus $\Pi_{d}$ in the form:

$$
\begin{aligned}
\left(\Pi_{u u}^{\tau s k}\right)_{11}= & n_{\alpha} C_{11}^{k} J_{\beta / \alpha}^{k \tau s} \partial_{\alpha}^{s}+n_{\beta} C_{66}^{k} J_{\alpha / \beta}^{k \tau s} \partial_{\beta}^{s} \\
& +n_{\beta} C_{16}^{k} J^{k \tau s} \partial_{\alpha}^{s}+n_{\alpha} C_{16}^{k} J^{k \tau s} \partial_{\beta}^{s}, \\
\left(\Pi_{u u}^{\tau s k}\right)_{12}= & n_{\alpha} C_{16}^{k} J_{\beta / \alpha}^{k \tau s} \partial_{\alpha}^{s}+n_{\beta} C_{26}^{k} J_{\alpha / \beta}^{k \tau s} \partial_{\beta}^{s} \\
& +n_{\alpha} C_{12}^{k} J^{k \tau s} \partial_{\beta}^{s}+n_{\beta} C_{66}^{k} J^{k \tau s} \partial_{\alpha}^{s}, \\
\left(\Pi_{u u}^{\tau s k}\right)_{13}= & n_{\alpha} \frac{1}{R_{\alpha k}} C_{11}^{k} J_{\beta / \alpha}^{k \tau s}+n_{\alpha} \frac{1}{R_{\beta k}} C_{12}^{k} J^{k \tau s} \\
& +n_{\alpha} C_{13}^{k} J_{\beta}^{k \tau s}+n_{\beta} \frac{1}{R_{\alpha k}} C_{16}^{k} J^{k \tau s} \\
& +n_{\beta} \frac{1}{R_{\beta k}} C_{26}^{k} J_{\alpha}^{k \tau s}+n_{\beta} C_{36}^{k} J_{\alpha}^{k \tau s_{z}}, \\
& +n_{\beta} C_{26}^{k} J^{k \tau s} \partial_{\alpha}^{s}+n_{\alpha} C_{26}^{k} J^{k \tau s} \partial_{\beta}^{s}, \\
& +n_{\beta} C_{12}^{k} J^{k \tau s} \partial_{\alpha}^{s}+n_{\alpha} C_{66}^{k} J^{k \tau s} \partial_{\beta}^{s}, \\
\left(\Pi_{u u}^{\tau s k}\right)_{21}= & n_{\alpha} C_{16}^{k} J_{\beta / \alpha}^{k \tau s} \partial_{\alpha}^{s}+n_{\beta} C_{26}^{k} J_{\alpha / \beta}^{k \tau s} \partial_{\beta}^{s} \\
\left(\Pi_{u u}^{\tau s k}\right)_{22}= & n_{\alpha} C_{66}^{k} J_{\beta / \alpha}^{k \tau s} \partial_{\alpha}^{s}+n_{\beta} C_{22}^{k} J_{\alpha / \beta}^{k \tau s} \partial_{\beta}^{s} \\
&
\end{aligned}
$$




$$
\begin{aligned}
\left(\Pi_{u u}^{\tau s k}\right)_{23}= & n_{\alpha} \frac{1}{R_{\alpha k}} C_{16}^{k} J_{\beta / \alpha}^{k \tau s}+n_{\alpha} \frac{1}{R_{\beta k}} C_{26}^{k} J^{k \tau s} \\
& +n_{\alpha} C_{36}^{k} J_{\beta}^{k \tau s_{z}}+n_{\beta} \frac{1}{R_{\alpha k}} C_{12}^{k} J^{k \tau s} \\
& +n_{\beta} \frac{1}{R_{\beta k}} C_{22}^{k} J_{\alpha / \beta}^{k \tau s}+n_{\beta} C_{23}^{k} J_{\alpha}^{k \tau s_{z}}, \\
\left(\Pi_{u u}^{\tau s k}\right)_{31}= & -n_{\alpha} \frac{1}{R_{\alpha k}} C_{55}^{k} J_{\beta / \alpha}^{k \tau s}+n_{\alpha} C_{55}^{k} J_{\beta}^{k \tau s_{z}} \\
& -n_{\beta} \frac{1}{R_{\alpha k}} C_{45}^{k} J^{k \tau s}+n_{\beta} C_{45}^{k} J_{\alpha}^{k \tau s_{z}}, \\
\left(\Pi_{u u}^{\tau s k}\right)_{32}= & -n_{\alpha} \frac{1}{R_{\beta k}} C_{45}^{k} J^{k \tau s}+n_{\alpha} C_{45}^{k} J_{\beta}^{k \tau s_{z}} \\
& -n_{\beta} \frac{1}{R_{\beta k}} C_{44}^{k} J_{\alpha / \beta}^{k \tau s}+n_{\beta} C_{44}^{k} J_{\alpha}^{k \tau s_{z}}, \\
\left(\Pi_{u u}^{\tau s k}\right)_{33}= & n_{\alpha} C_{55}^{k} J_{\beta / \alpha}^{k \tau s} \partial_{\alpha}^{s}+n_{\beta} C_{44}^{k} J_{\alpha / \beta}^{k \tau s} \partial_{\beta}^{s} \\
& +n_{\beta} C_{45}^{k} J^{k \tau s} \partial_{\alpha}^{s}+n_{\alpha} C_{45}^{k} J^{k \tau s} \partial_{\beta}^{s} .
\end{aligned}
$$

One can note that all the equations written for the shell degenerate into those for the plate when $1 / R_{\alpha k}=1 / R_{\beta k}=0$. In practice we set the radii of curvature to $10^{9}$.

2.4. Dynamic Governing Equations. The PVD for the dynamic case is expressed as

$$
\begin{array}{r}
\sum_{k=1}^{N_{l}} \int_{\Omega_{k}} \int_{A_{k}}\left\{\delta \boldsymbol{\epsilon}_{p G}^{k T} \boldsymbol{\sigma}_{p C}^{k}+\delta \boldsymbol{\epsilon}_{n G}^{k T} \boldsymbol{\sigma}_{n C}^{k}\right\} d \Omega_{k} d z \\
=\sum_{k=1}^{N_{l}} \int_{\Omega_{k}} \int_{A_{k}} \rho^{k} \delta \mathbf{u}^{k T} \ddot{\mathbf{u}}^{k} d \Omega_{k} d z+\sum_{k=1}^{N_{l}} \delta L_{e}^{k},
\end{array}
$$

where $\rho^{k}$ is the mass density of the $k$ th layer and double dots denote acceleration.

By substituting the geometrical relations, the constitutive equations, and the Unified Formulation, we obtain the following governing equations:

$$
\delta \mathbf{u}_{s}^{k^{T}}: \mathbf{K}_{u u}^{k \tau s} \mathbf{u}_{\tau}^{k}=\mathbf{M}^{k \tau s} \ddot{\mathbf{u}}_{\tau}^{k}+\mathbf{P}_{u \tau}^{k} .
$$

In the case of free vibrations one has

$$
\delta \mathbf{u}_{s}^{k^{T}}: \mathbf{K}_{u u}^{k \tau s} \mathbf{u}_{\tau}^{k}=\mathbf{M}^{k \tau s} \ddot{\mathbf{u}}_{\tau}^{k},
$$

where $\mathbf{M}^{k \tau s}$ is the fundamental nucleus for the inertial term. The explicit form of that is

$$
\begin{aligned}
& \mathbf{M}_{11}^{k \tau s}=\rho^{k} J_{\alpha \beta}^{k \tau s}, \quad \mathbf{M}_{12}^{k \tau s}=0, \quad \mathbf{M}_{13}^{k \tau s}=0, \\
& \mathbf{M}_{21}^{k \tau s}=0, \quad \mathbf{M}_{22}^{k \tau s}=\rho^{k} J_{\alpha \beta}^{k \tau s}, \quad \mathbf{M}_{23}^{k \tau s}=0, \\
& \mathbf{M}_{31}^{k \tau s}=0, \quad \mathbf{M}_{32}^{k \tau s}=0, \quad \mathbf{M}_{33}^{k \tau s}=\rho^{k} J_{\alpha \beta}^{k \tau s},
\end{aligned}
$$

where the meaning of the integral $J_{\alpha \beta}^{k \tau s}$ has been illustrated in (25). The geometrical and mechanical boundary conditions are the same of the static case. Because we consider the static case only, the mass terms will be neglected.

\section{The Radial Basis Function Method}

3.1. The Static Problem. Radial basis functions (RBFs) approximations are mesh-free numerical schemes that can exploit accurate representations of the boundary, are easy to implement, and can be spectrally accurate. In this section the formulation of a global unsymmetrical collocation RBFbased method to compute elliptic operators is presented.

Consider a linear elliptic partial differential operator $L$ and a bounded region $\Omega$ in $\mathbb{R}^{n}$ with some boundary $\partial \Omega$. In the static problems we seek the computation of displacement (u) from the global system of equations

$$
\begin{gathered}
\mathscr{L} \mathbf{u}=\mathbf{f} \text { in } \Omega, \\
\mathscr{L}_{B} \mathbf{u}=\mathbf{g} \text { on } \partial \Omega,
\end{gathered}
$$

where $\mathscr{L}$ and $\mathscr{L}_{B}$ are linear operators in the domain and on the boundary, respectively. The right-hand sides of (32) represent the external forces applied on the plate or shell and the boundary conditions applied along the perimeter of the plate or shell, respectively. The PDE problem defined in (32) will be replaced by a finite problem, defined by an algebraic system of equations, after the radial basis expansions.

3.2. The Eigenproblem. The eigenproblem looks for eigenvalues $(\lambda)$ and eigenvectors (u) that satisfy

$$
\begin{aligned}
& \mathscr{L} \mathbf{u}+\lambda \mathbf{u}=0 \text { in } \Omega, \\
& \mathscr{L}_{B} \mathbf{u}=0 \text { on } \partial \Omega .
\end{aligned}
$$

As in the static problem, the eigenproblem defined in (33) is replaced by a finite-dimensional eigenvalue problem, based on RBF approximations.

3.3. Radial Basis Functions Approximations. The radial basis function $(\phi)$ approximation of a function ( $\mathbf{u})$ is given by

$$
\widetilde{\mathbf{u}}(\mathbf{x})=\sum_{i=1}^{N} \alpha_{i} \phi\left(\left\|x-y_{i}\right\|_{2}\right), \quad \mathbf{x} \in \mathbb{R}^{n},
$$

where $y_{i}, i=1, \ldots, N$, is a finite set of distinct points (centers) in $\mathbb{R}^{n}$. The most common RBFs are

$$
\text { Cubic: } \phi(r)=r^{3} \text {, }
$$

Thin plate splines: $\phi(r)=r^{2} \log (r)$,

Wendland functions: $\phi(r)=(1-r)_{+}^{m} p(r)$,

$$
\text { Gaussian: } \phi(r)=e^{-(c r)^{2}} \text {, }
$$

Multiquadrics: $\phi(r)=\sqrt{c^{2}+r^{2}}$,

Inverse Multiquadrics: $\phi(r)=\left(c^{2}+r^{2}\right)^{-1 / 2}$, 
where the Euclidian distance $r$ is real and nonnegative and $c$ is a positive shape parameter. Hardy [54] introduced multiquadrics in the analysis of scattered geographical data. In the 1990s Kansa [31] used multiquadrics for the solution of partial differential equations. Considering $N$ distinct interpolations and knowing $u\left(x_{j}\right), j=1,2, \ldots, N$, we find $\alpha_{i}$ by the solution of a $N \times N$ linear system

$$
\mathbf{A} \alpha=\mathbf{u}
$$

where $\mathbf{A}=\left[\phi\left(\left\|x-y_{i}\right\|_{2}\right)\right]_{N \times N}, \boldsymbol{\alpha}=\left[\alpha_{1}, \alpha_{2}, \ldots, \alpha_{N}\right]^{T}$, and $\mathbf{u}=\left[u\left(x_{1}\right), u\left(x_{2}\right), \ldots, u\left(x_{N}\right)\right]^{T}$.

3.4. Solution of the Static Problem. The solution of a static problem by radial basis functions considers $N_{I}$ nodes in the domain and $N_{B}$ nodes on the boundary, with a total number of nodes $N=N_{I}+N_{B}$. We denote the sampling points by $x_{i} \in \Omega, i=1, \ldots, N_{I}$, and $x_{i} \in \partial \Omega, i=N_{I}+1, \ldots, N$. At the points in the domain we solve the following system of equations:

$$
\sum_{i=1}^{N} \alpha_{i} \mathscr{L} \phi\left(\left\|x-y_{i}\right\|_{2}\right)=\mathbf{f}\left(x_{j}\right), \quad j=1,2, \ldots, N_{I},
$$

or

$$
\mathscr{L}^{I} \boldsymbol{\alpha}=\mathbf{F}
$$

where

$$
\mathscr{L}^{I}=\left[\mathscr{L} \phi\left(\left\|x-y_{i}\right\|_{2}\right)\right]_{N_{I} \times N^{*}}
$$

At the points on the boundary, we impose boundary conditions as

$$
\sum_{i=1}^{N} \alpha_{i} \mathscr{L}_{B} \phi\left(\left\|x-y_{i}\right\|_{2}\right)=\mathbf{g}\left(x_{j}\right), \quad j=N_{I}+1, \cdots, N,
$$

or

$$
\mathrm{B} \boldsymbol{\alpha}=\mathrm{G}
$$

where

$$
\mathbf{B}=\mathscr{L}_{B} \phi\left[\left(\left\|x_{N_{I}+1}-y_{j}\right\|_{2}\right)\right]_{N_{B} \times N} .
$$

Therefore, we can write a finite-dimensional static problem as

$$
\left[\begin{array}{c}
\mathscr{L}^{I} \\
\mathbf{B}
\end{array}\right] \boldsymbol{\alpha}=\left[\begin{array}{l}
\mathbf{F} \\
\mathbf{G}
\end{array}\right]
$$

By inverting the system (43), we obtain the vector $\boldsymbol{\alpha}$. We then obtain the solution $\mathbf{u}$ using the interpolation (34).

3.5. Solution of the Eigenproblem. We consider $N_{I}$ nodes in the interior of the domain and $N_{B}$ nodes on the boundary, with $N=N_{I}+N_{B}$. We denote interpolation points by $x_{i} \in \Omega$, $i=1, \ldots, N_{I}$, and $x_{i} \in \partial \Omega, i=N_{I}+1, \ldots, N$. At the points in the domain, we define the eigenproblem as

$$
\sum_{i=1}^{N} \alpha_{i} \mathscr{L} \phi\left(\left\|x-y_{i}\right\|_{2}\right)=\lambda \widetilde{\mathbf{u}}\left(x_{j}\right), \quad j=1,2, \ldots, N_{I},
$$

or

$$
\mathscr{L}^{I} \boldsymbol{\alpha}=\lambda \widetilde{\mathbf{u}}^{I}
$$

where

$$
\mathscr{L}^{I}=\left[\mathscr{L} \phi\left(\left\|x-y_{i}\right\|_{2}\right)\right]_{N_{I} \times N^{*}}
$$

At the points on the boundary, we enforce the boundary conditions as

$$
\sum_{i=1}^{N} \alpha_{i} \mathscr{L}_{B} \phi\left(\left\|x-y_{i}\right\|_{2}\right)=0, \quad j=N_{I}+1, \ldots, N,
$$

or

$$
\mathbf{B} \boldsymbol{\alpha}=0 .
$$

Equations (45) and (48) can now be solved as a generalized eigenvalue problem

$$
\left[\begin{array}{c}
\mathscr{L}^{I} \\
\mathbf{B}
\end{array}\right] \boldsymbol{\alpha}=\lambda\left[\begin{array}{c}
\mathbf{A}^{I} \\
\mathbf{0}
\end{array}\right] \boldsymbol{\alpha}
$$

where

$$
\mathbf{A}^{I}=\phi\left[\left(\left\|x_{N_{I}}-y_{j}\right\|_{2}\right)\right]_{N_{I} \times N} .
$$

3.6. Discretization of the Equations of Motion and Boundary Conditions. The radial basis collocation method follows a simple implementation procedure. Taking (11), we compute

$$
\boldsymbol{\alpha}=\left[\begin{array}{c}
L^{I} \\
\mathbf{B}
\end{array}\right]^{-1}\left[\begin{array}{l}
\mathbf{F} \\
\mathbf{G}
\end{array}\right]
$$

This $\boldsymbol{\alpha}$ vector is then used to obtain solution $\widetilde{\mathbf{u}}$, by using (5). If derivatives of $\widetilde{\mathbf{u}}$ are needed, such derivatives are computed as

$$
\begin{gathered}
\frac{\partial \widetilde{\mathbf{u}}}{\partial x}=\sum_{j=1}^{N} \alpha_{j} \frac{\partial \phi_{j}}{\partial x}, \\
\frac{\partial^{2} \widetilde{\mathbf{u}}}{\partial x^{2}}=\sum_{j=1}^{N} \alpha_{j} \frac{\partial^{2} \phi_{j}}{\partial x^{2}},
\end{gathered}
$$

and so forth.

In the present collocation approach, we need to impose essential and natural boundary conditions. Consider, for example, the condition $w=0$, on a simply supported or clamped edge. We enforce the conditions by interpolating as

$$
w=0 \longrightarrow \sum_{j=1}^{N} \alpha_{j}^{W} \phi_{j}=0 .
$$

Other boundary conditions are interpolated in a similar way.

3.7. Free Vibrations Problems. For free vibration problems we set the external force to zero and assume harmonic solution in terms of displacement, $u_{0}, u_{1}, v_{0}, v_{1}, \ldots$, as

$$
\begin{gathered}
u_{0}=U_{0}(w, y) e^{i \omega t} ; \quad u_{1}=U_{1}(w, y) e^{i \omega t}, \\
u_{Z}=U_{Z}(w, y) e^{i \omega t} \\
v_{0}=V_{0}(w, y) e^{i \omega t}, \quad v_{1}=V_{1}(w, y) e^{i \omega t},
\end{gathered}
$$


TABLE 1: Nondimensional central deflection, $\bar{w}=w\left(10^{2} E_{2} h^{3} / P_{0} a^{4}\right)$, and variation with various numbers of grid points per unit length, $N$, for different $R / a$ ratios, for $R_{1}=R_{2}$.

\begin{tabular}{|c|c|c|c|c|c|c|c|c|}
\hline & $a / h$ & Method & & & & & & \\
\hline & $u / n$ & DVCtIIU & 5 & 10 & 20 & 50 & 100 & $10^{9}$ \\
\hline & 10 & Present $(13 \times 13)$ & 6.8510 & 7.0818 & 7.1429 & 7.1609 & 7.1637 & 7.1649 \\
\hline & 10 & Present $(17 \times 17)$ & 6.8516 & 7.0822 & 7.1433 & 7.1612 & 7.1640 & 7.1653 \\
\hline & 10 & Present $(21 \times 21)$ & 6.8516 & 7.0822 & 7.1433 & 7.1612 & 7.1640 & 7.1653 \\
\hline & 10 & HSDT [56] & 6.7688 & 7.0325 & 7.1016 & 7.1212 & 7.1240 & 7.125 \\
\hline & 10 & FSDT [56] & 6.4253 & 6.6247 & 6.6756 & 6.6902 & 6.6923 & 6.6939 \\
\hline$\left[0^{\circ} / 90^{\circ} / 0^{\circ}\right]$ & 100 & Present $(13 \times 13)$ & 1.0245 & 2.3658 & 3.5167 & 4.0714 & 4.1652 & 4.1975 \\
\hline & 100 & Present $(17 \times 17)$ & 1.0250 & 2.3667 & 3.5181 & 4.0728 & 4.1667 & 4.1990 \\
\hline & 100 & Present $(21 \times 21)$ & 1.0250 & 2.3669 & 3.5183 & 4.0731 & 4.1669 & 4.1992 \\
\hline & 100 & HSDT [56] & 1.0321 & 2.4099 & 3.617 & 4.2071 & 4.3074 & 4.3420 \\
\hline & 100 & FSDT [56] & 1.0337 & 2.4109 & 3.6150 & 4.2027 & 4.3026 & 4.3370 \\
\hline & 10 & Present $(13 \times 13)$ & 6.7737 & 7.0012 & 7.0614 & 7.0791 & 7.0819 & 7.0831 \\
\hline & 10 & Present $(17 \times 17)$ & 6.7742 & 7.0015 & 7.0618 & 7.0795 & 7.0822 & 7.0834 \\
\hline & 10 & Present $(21 \times 21)$ & 6.7742 & 7.0015 & 7.0618 & 7.0795 & 7.0822 & 7.0834 \\
\hline & 10 & HSDT [56] & 6.7865 & 7.0536 & 7.1237 & 7.1436 & 7.1464 & 7.1474 \\
\hline & 10 & FSDT [56] & 6.3623 & 6.5595 & 6.6099 & 6.6244 & 6.6264 & 6.6280 \\
\hline$\left[0^{\circ} / 90^{\circ} / 90^{\circ} / 0^{\circ}\right]$ & 100 & Present $(13 \times 13)$ & 1.0190 & 2.3583 & 3.5125 & 4.0702 & 4.1647 & 4.1972 \\
\hline & 100 & Present $(17 \times 17)$ & 1.0194 & 2.3593 & 3.5138 & 4.0717 & 4.1662 & 4.1987 \\
\hline & 100 & Present $(21 \times 21)$ & 1.0195 & 2.3594 & 3.5140 & 4.0719 & 4.1664 & 4.1989 \\
\hline & 100 & HSDT [56] & 1.0264 & 2.4024 & 3.6133 & 4.2071 & 4.3082 & 4.3430 \\
\hline & 100 & FSDT [56] & 1.0279 & 2.4030 & 3.6104 & 4.2015 & 4.3021 & 4.3368 \\
\hline
\end{tabular}

$$
\begin{gathered}
v_{Z}=V_{Z}(w, y) e^{i \omega t} \\
w_{0}=W_{0}(w, y) e^{i \omega t}, \quad w_{1}=W_{1}(w, y) e^{i \omega t}, \\
w_{2}=W_{2}(w, y) e^{i \omega t},
\end{gathered}
$$

where $\omega$ is the frequency of natural vibration. Substituting the harmonic expansion into (49) in terms of the amplitudes $U_{0}, U_{1}, U_{Z}, V_{0}, V_{1}, V_{Z}, W_{0}, W_{1}$, and $W_{2}$, we may obtain the natural frequencies and vibration modes for the plate or shell problem, by solving the eigenproblem

$$
\left[\mathscr{L}-\omega^{2} \mathscr{G}\right] \mathbf{X}=\mathbf{0}
$$

where $\mathscr{L}$ collects all stiffness terms and $\mathscr{G}$ collects all terms related to the inertial terms. In (55) $\mathbf{X}$ are the modes of vibration associated with the natural frequencies defined as $\omega$.

\section{Numerical Examples}

All numerical examples consider a Chebyshev grid and a Wendland function, defined as

$$
\phi(r)=(1-c r)^{8}+\left(32(c r)^{3}+25(c r)^{2}+8 c r+1\right),
$$

where the shape parameter $(c)$ was obtained by an optimization procedure, as detailed in Ferreira and Fasshauer [55].

4.1. Spherical Shell in Bending. A laminated composite spherical shell is here considered, of side $a$ and thickness $h$, to be composed of layers oriented at $\left[0^{\circ} / 90^{\circ} / 0^{\circ}\right]$ and $\left[0^{\circ} / 90^{\circ} / 90^{\circ} / 0^{\circ}\right]$. The shell is subjected to a sinusoidal vertical pressure of the form

$$
p_{z}=P \sin \left(\frac{\pi x}{a}\right) \sin \left(\frac{\pi y}{a}\right)
$$

with the origin of the coordinate system located at the lower left corner on the midplane and $P$ the maximum load (at center of shell).

The orthotropic material properties for each layer are given by

$$
\begin{gathered}
E_{1}=25.0 E_{2}, \quad G_{12}=G_{13}=0.5 E_{2}, \\
G_{23}=0.2 E_{2}, \quad v_{12}=0.25 .
\end{gathered}
$$

The in-plane displacement, the transverse displacement, the normal stresses, and the in-plane and transverse shear stresses are presented in normalized form as

$$
\begin{gathered}
2 \bar{w}=\frac{10^{2} w_{(a / 2, a / 2,0)} h^{3} E_{2}}{P a^{4}}, \quad \bar{\sigma}_{x x}=\frac{\sigma_{x x(a / 2, a / 2, h / 2)} h^{2}}{P a^{2}}, \\
\bar{\sigma}_{y y}=\frac{\sigma_{y y(a / 2, a / 2, h / 4)} h^{2}}{P a^{2}}, \quad \bar{\tau}_{x z}=\frac{\tau_{x z(0, a / 2,0)} h}{P a}, \\
\bar{\tau}_{x y}=\frac{\tau_{x y(0,0, h / 2)} h^{2}}{P a^{2}} .
\end{gathered}
$$

The shell is simply supported on all edges.

In Table 1 we compare the static deflections for the present shell model with results of Reddy shell formulation using 


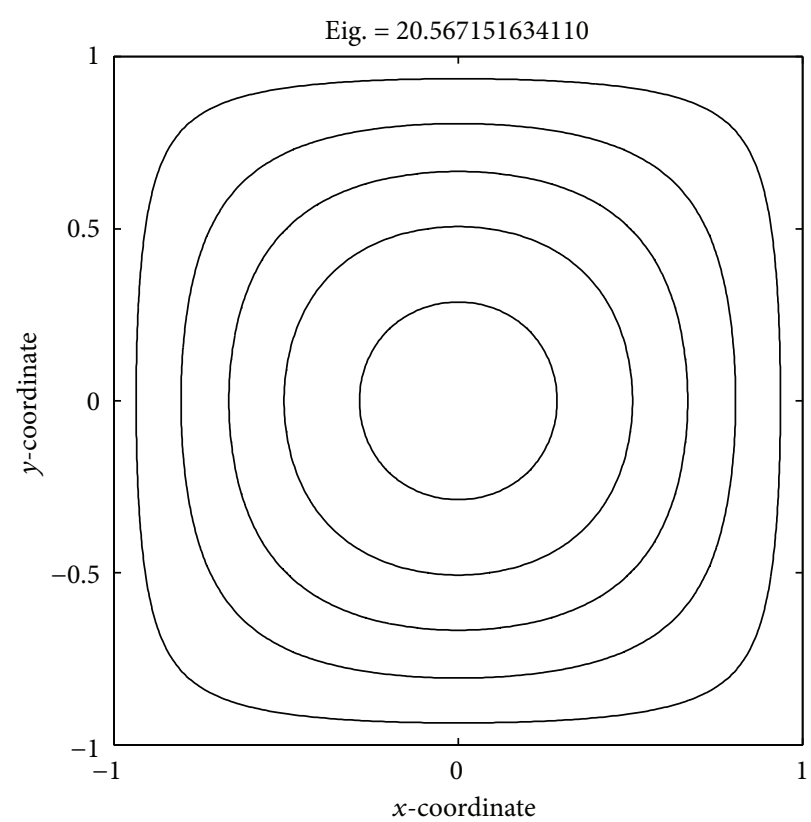

(a)

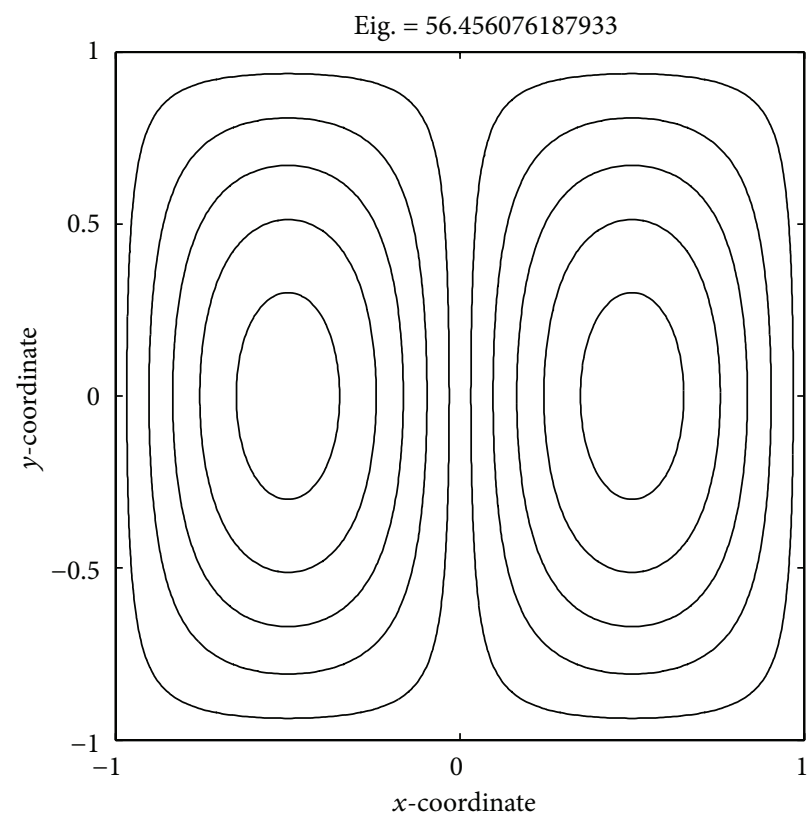

(c)

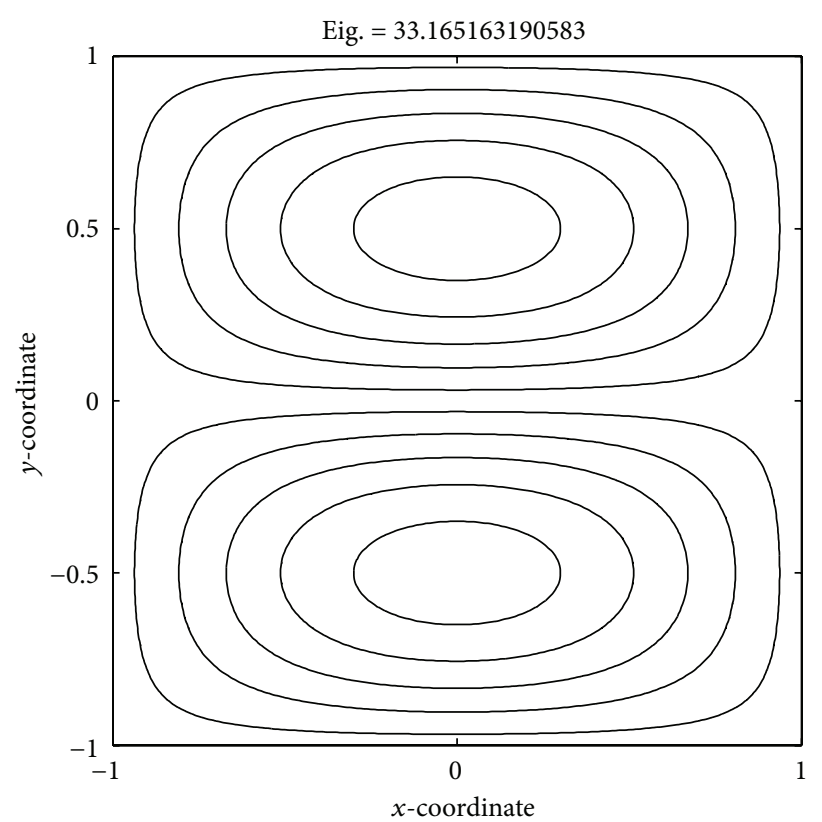

(b)

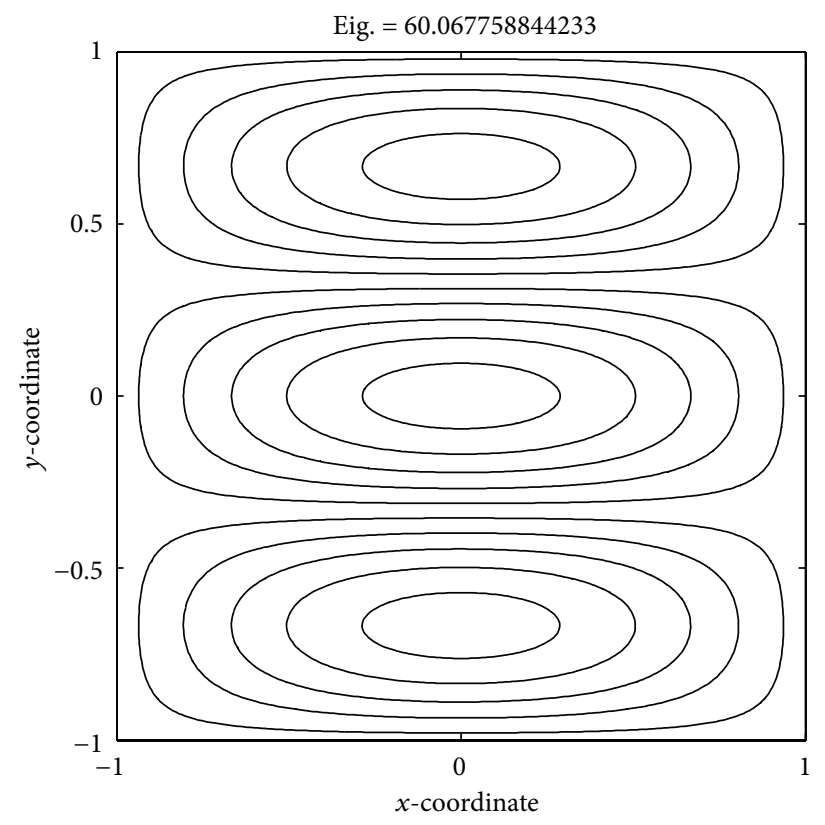

(d)

Figure 4: The first 4 vibrational modes of cross-ply laminated spherical shells, $\bar{\omega}=\omega\left(a^{2} / h\right) \sqrt{\rho / E_{2}}$, for laminate $\left(\left[0^{\circ} / 90^{\circ} / 90^{\circ} / 0^{\circ}\right]\right)$ using a grid of $21 \times 21$ points, for $a / h=100, R / a=10$.

first-order and third-order shear deformation theories [56]. We consider nodal grids with $13 \times 13,17 \times 17$, and $21 \times 21$ points. We consider various values of $R / a$ and two values of $a / h$ (10 and 100). Results are in good agreement for various $a / h$ ratios with the higher-order results of Reddy and Liu [56].

4.2. Free Vibration of Spherical and Cylindrical Laminated Shells. We consider nodal grids with $13 \times 13,17 \times 17$, and
$21 \times 21$ points. In Tables 2 and 3 we compare the nondimensionalized natural frequencies from the present Murakami theory for various cross-ply spherical shells, with analytical solutions done by Reddy and Liu [56] who considered both the first-order (FSDT) and the third-order (HSDT) theories. The first-order theory overpredicts the fundamental natural frequencies of symmetric thick shells and symmetric shallow thin shells. The present radial basis function method is 
TABLE 2: Nondimensionalized fundamental frequencies of cross-ply laminated spherical shells, $\bar{\omega}=\omega\left(a^{2} / h\right) \sqrt{\rho / E_{2}}$, and laminate $\left(\left[0^{\circ} / 90^{\circ} / 90^{\circ} / 0^{\circ}\right]\right)$.

\begin{tabular}{|c|c|c|c|c|c|c|c|}
\hline \multirow{2}{*}{$a / h$} & \multirow{2}{*}{ Method } & \multicolumn{6}{|c|}{$R / a$} \\
\hline & & 5 & 10 & 20 & 50 & 100 & $10^{9}$ \\
\hline \multirow{4}{*}{10} & Present $(13 \times 13)$ & 12.0527 & 11.8889 & 11.8474 & 11.8357 & 11.8340 & 11.8335 \\
\hline & Present $(17 \times 17)$ & 12.0523 & 11.8886 & 11.8471 & 11.8355 & 11.8338 & 11.8332 \\
\hline & Present $(21 \times 21)$ & 12.0522 & 11.8885 & 11.8470 & 11.8355 & 11.8338 & 11.8332 \\
\hline & HSDT [56] & 12.040 & 11.840 & 11.790 & 11.780 & 11.780 & 11.780 \\
\hline \multirow{4}{*}{100} & Present $(13 \times 13)$ & 31.2170 & 20.5742 & 16.8698 & 15.6744 & 15.4960 & 15.4361 \\
\hline & Present $(17 \times 17)$ & 31.2072 & 20.5679 & 16.8648 & 15.6698 & 15.4915 & 15.4316 \\
\hline & Present $(21 \times 21)$ & 31.2059 & 20.5672 & 16.8642 & 15.6693 & 15.4910 & 15.4311 \\
\hline & HSDT [56] & 31.100 & 20.380 & 16.630 & 15.420 & 15.230 & 15.170 \\
\hline
\end{tabular}

TABLE 3: Nondimensionalized fundamental frequencies of cross-ply laminated spherical shells, $\bar{\omega}=\omega\left(a^{2} / h\right) \sqrt{\rho / E_{2}}$, and laminate $\left(\left[0^{\circ} / 90^{\circ} / 0^{\circ}\right]\right)$.

\begin{tabular}{|c|c|c|c|c|c|c|c|}
\hline \multirow{2}{*}{$a / h$} & \multirow{2}{*}{ Method } & \multicolumn{6}{|c|}{$R / a$} \\
\hline & & 5 & 10 & 20 & 50 & 100 & $10^{9}$ \\
\hline \multirow{4}{*}{10} & Present $(13 \times 13)$ & 11.9831 & 11.8192 & 11.7777 & 11.7660 & 11.7643 & 11.7638 \\
\hline & Present $(17 \times 17)$ & 11.9827 & 11.8190 & 11.7774 & 11.7658 & 11.7641 & 11.7635 \\
\hline & Present $(21 \times 21)$ & 11.9827 & 11.8190 & 11.7774 & 11.7658 & 11.7641 & 11.7635 \\
\hline & HSDT [56] & 12.060 & 11.860 & 11.810 & 11.790 & 11.790 & 11.790 \\
\hline \multirow{4}{*}{100} & Present $(13 \times 13)$ & 31.1343 & 20.5420 & 16.8595 & 15.6721 & 15.4950 & 15.4355 \\
\hline & Present $(17 \times 17)$ & 31.1244 & 20.5357 & 16.8545 & 15.6675 & 15.4905 & 15.4310 \\
\hline & Present $(21 \times 21)$ & 31.1231 & 20.5350 & 16.8540 & 15.6671 & 15.4901 & 15.4306 \\
\hline & HSDT [56] & 31.020 & 20.350 & 16.620 & 15.420 & 15.240 & 15.170 \\
\hline
\end{tabular}

TABLE 4: Nondimensionalized fundamental frequencies of cross-ply cylindrical shells, $\bar{\omega}=\omega\left(a^{2} / h\right) \sqrt{\rho / E_{2}}$.

\begin{tabular}{|c|c|c|c|c|c|}
\hline$R / a$ & Method & $\begin{array}{c}{[0 / 90 / 0]} \\
a / h=100\end{array}$ & $a / h=10$ & $\begin{array}{c}{[0 / 90 / 90 / 0]} \\
a / h=100\end{array}$ & $a / h=10$ \\
\hline \multirow{5}{*}{5} & Present $(13 \times 13)$ & 20.4956 & 11.7774 & 20.5298 & 11.8524 \\
\hline & Present $(17 \times 17)$ & 20.4839 & 11.7770 & 20.5201 & 11.8521 \\
\hline & Present $(21 \times 21)$ & 20.4829 & 11.7770 & 20.5189 & 11.8521 \\
\hline & FSDT [56] & 20.332 & 12.207 & 20.361 & 12.267 \\
\hline & HSDT [56] & 20.330 & 11.850 & 20.360 & 11.830 \\
\hline \multirow{5}{*}{10} & Present $(13 \times 13)$ & 16.8475 & 11.7672 & 16.8583 & 11.8382 \\
\hline & Present $(17 \times 17)$ & 16.8409 & 11.7669 & 16.8522 & 11.8380 \\
\hline & Present $(21 \times 21)$ & 16.8401 & 11.7669 & 16.8516 & 11.8380 \\
\hline & FSDT [56] & 16.625 & 12.173 & 16.634 & 12.236 \\
\hline & HSDT [56] & 16.620 & 11.800 & 16.630 & 11.790 \\
\hline \multirow{5}{*}{20} & Present $(13 \times 13)$ & 15.8006 & 11.7646 & 15.8039 & 11.8346 \\
\hline & Present $(17 \times 17)$ & 15.7955 & 11.7644 & 15.7990 & 11.8344 \\
\hline & Present $(21 \times 21)$ & 15.7950 & 11.7644 & 15.7985 & 11.8344 \\
\hline & FSDT [56] & 15.556 & 12.166 & 15.559 & 12.230 \\
\hline & HSDT [56] & 15.55 & 11.79 & 15.55 & 11.78 \\
\hline \multirow{5}{*}{50} & Present $(13 \times 13)$ & 15.4945 & 11.7639 & 15.4955 & 11.8336 \\
\hline & Present $(17 \times 17)$ & 15.4899 & 11.7637 & 15.4909 & 11.8334 \\
\hline & Present $(21 \times 21)$ & 15.4895 & 11.7637 & 15.4905 & 11.8334 \\
\hline & FSDT [56] & 15.244 & 12.163 & 15.245 & 12.228 \\
\hline & HSDT [56] & 15.24 & 11.79 & 15.23 & 11.78 \\
\hline \multirow{5}{*}{100} & Present $(13 \times 13)$ & 15.4503 & 11.7638 & 15.4510 & 11.8335 \\
\hline & Present $(17 \times 17)$ & 15.4457 & 11.7636 & 15.4464 & 11.8333 \\
\hline & Present $(21 \times 21)$ & 15.4453 & 11.7636 & 15.4460 & 11.8333 \\
\hline & FSDT [56] & 15.198 & 12.163 & 15.199 & 12.227 \\
\hline & HSDT [56] & 15.19 & 11.79 & 15.19 & 11.78 \\
\hline \multirow{5}{*}{ Plate } & Present $(13 \times 13)$ & 15.4355 & 11.7638 & 15.4361 & 11.8335 \\
\hline & Present $(17 \times 17)$ & 15.4310 & 11.7635 & 15.4316 & 11.8332 \\
\hline & Present $(21 \times 21)$ & 15.4306 & 11.7635 & 15.4311 & 11.8332 \\
\hline & FSDT $[56]$ & 15.183 & 12.162 & 15.184 & 12.226 \\
\hline & HSDT [56] & 15.170 & 11.790 & 15.170 & 11.780 \\
\hline
\end{tabular}




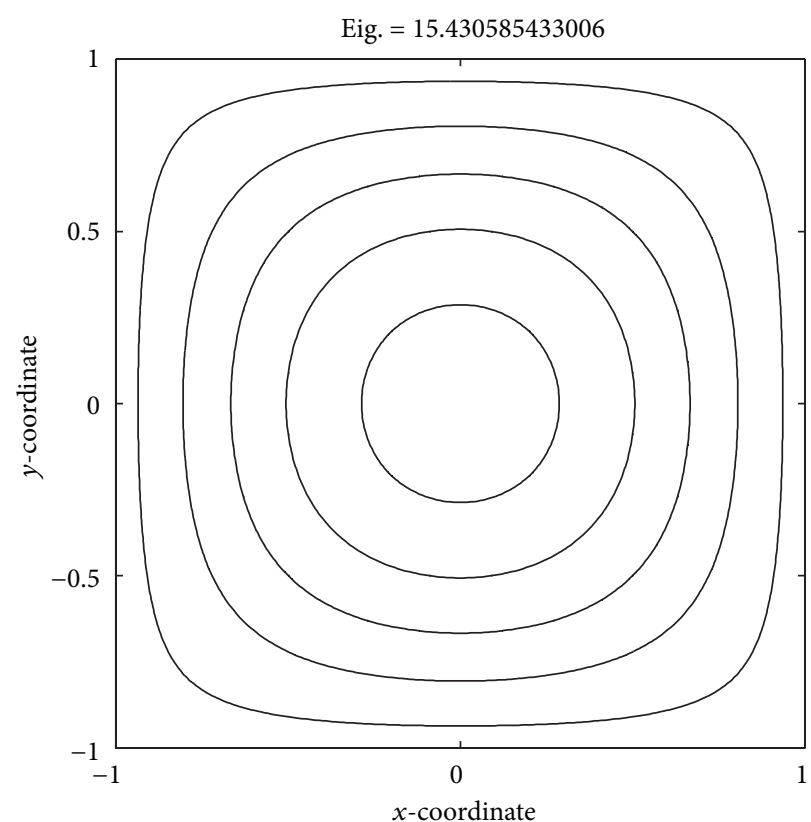

(a)

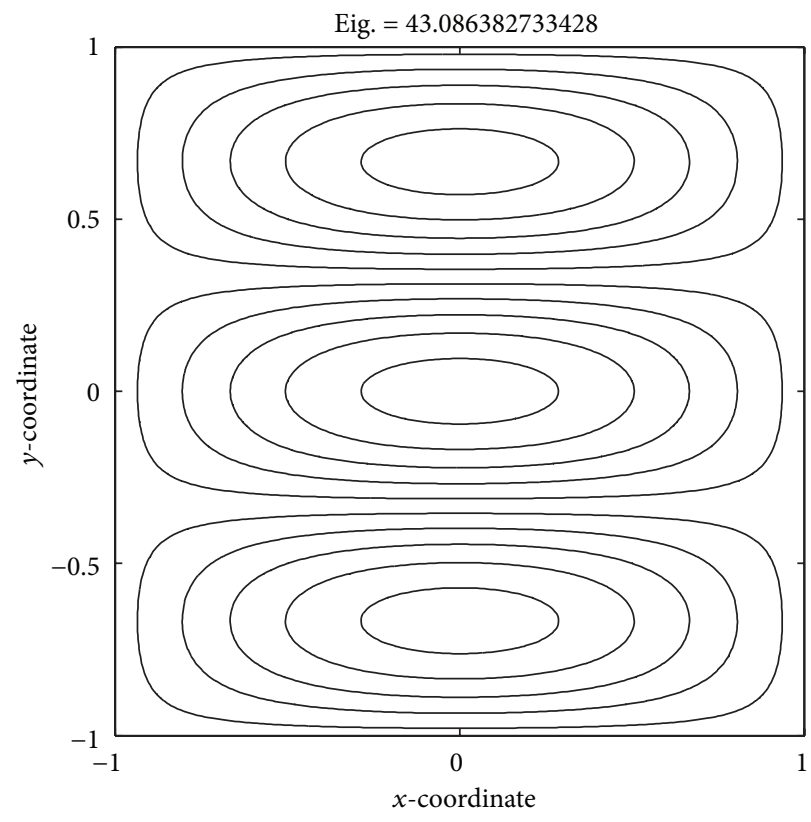

(c)

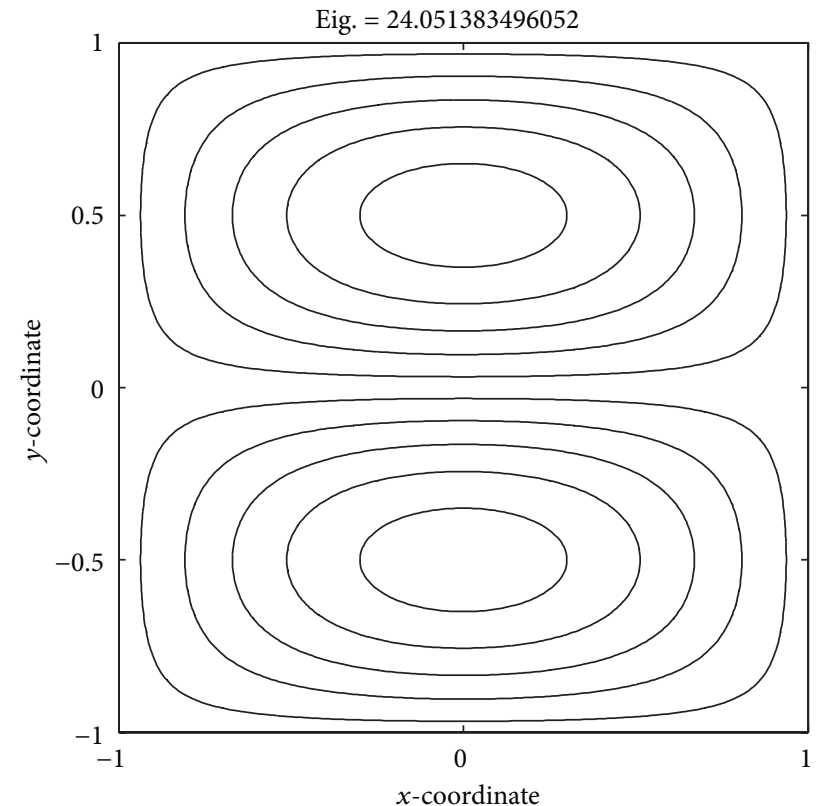

(b)

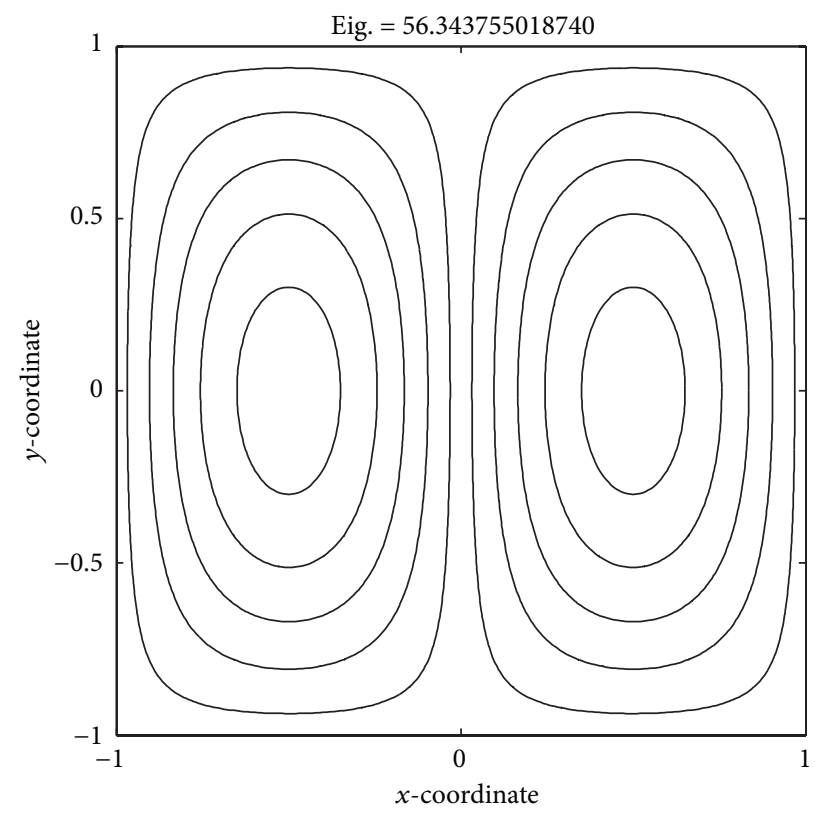

(d)

FIGURE 5: The first 4 vibrational modes of cross-ply laminated spherical shells, $\bar{\omega}=\omega\left(a^{2} / h\right) \sqrt{\rho / E_{2}}$, for laminate $\left(\left[0^{\circ} / 90^{\circ} / 0^{\circ}\right]\right)$ using a grid of $21 \times 21$ points, for $a / h=100, R / a=10 e^{9}$ (plate).

compared with analytical results by Reddy and Liu [56] and shows excellent agreement.

Table 4 contains nondimensionalized natural frequencies obtained using the the present Murakami theory for crossply cylindrical shells with lamination schemes [0/90/0], [0/90/90/0]. Present results are compared with analytical solutions done by Reddy and Liu [56] who considered both the first-order (FSDT) and the third-order (HSDT) theories. The present radial basis function method is compared with analytical results by Reddy and Liu [56] and shows excellent agreement.

In Figure 4 we illustrate the first 4 vibrational modes of cross-ply laminated spherical shells, $\bar{\omega}=\omega\left(a^{2} / h\right) \sqrt{\rho / E_{2}}$, for a laminate $\left(\left[0^{\circ} / 90^{\circ} / 90^{\circ} / 0^{\circ}\right]\right)$, using a grid of $21 \times 21$ points, for $a / h=100, R / a=10$. The modes of vibration are quite stable.

In Figure 5 we illustrate the first 4 vibrational modes of cross-ply laminated spherical shells, $\bar{\omega}=\omega\left(a^{2} / h\right) \sqrt{\rho / E_{2}}$, for a laminate $\left(\left[0^{\circ} / 90^{\circ} / 0^{\circ}\right]\right)$, using a grid of $21 \times 21$ points, for 
$a / h=100, R / a=10 e^{9}$. Again the modes of vibration are quite stable.

\section{Concluding Remarks}

In this paper Murakami's theory was implemented for the first time for laminated orthotropic elastic shells through a multiquadric discretization of equations of motion and boundary conditions. The multiquadric radial basis function method for the solution of shell bending and free vibration problems was presented. Results for static deformations and natural frequencies were obtained and compared with other sources. This meshless approach demonstrated that is very successful in the static deformations and free vibration analysis of laminated composite shells. Advantages of radial basis functions are absence of mesh, ease of discretization of boundary conditions and equations of equilibrium or motion, and very easy coding. We show that the static displacement and stresses and the natural frequencies obtained from present method are in excellent agreement with analytical solutions.

\section{Conflict of Interests}

The authors declare that there is no conflict of interests regarding the publication of this paper.

\section{Acknowledgments}

This paper was funded by the Deanship of Scientific Research (DSR), King Abdulaziz University, Jeddah, under Grant no. HiCi/1433/363-4. The authors, therefore, acknowledge with thanks DSR technical and financial support.

\section{References}

[1] W. T. Koiter, "On the foundations of the linear theory of thin elastic shells," Koninklijke Nederlandse Akademie van Wetenschappen, vol. 73, pp. 169-195, 1970.

[2] P. M. Naghdi, "A survey of recent progress in the theory of elastic shells," Applied Mechanics Reviews, vol. 9, pp. 365-368, 1956.

[3] D. Zenkert, An Introduction to Sandwich Structures, Chamelon Press, Oxford, UK, 1995.

[4] J. R. Vinson, The Behavior of Sandwich Structures of Isotropic and Composite Materials, Technomic Publishing Co., 1999.

[5] W. S. Burton and A. K. Noor, "Assessment of computational models for sandwich panels and shells," Computer Methods in Applied Mechanics and Engineering, vol. 124, no. 1-2, pp. 125-151, 1995.

[6] A. K. Noor, W. S. Burton, and C. W. Bert, "Computational models for sandwich panels and shells," Applied Mechanics Reviews, vol. 49, no. 3, pp. 155-199, 1996.

[7] H. Altenbach, "Theories for laminated and sandwich plates. A review," Mechanics of Composite Materials, vol. 34, no. 3, pp. 243-252, 1998.

[8] L. Librescu and T. Hause, "Recent developments in the modeling and behavior of advanced sandwich constructions: a survey," Composite Structures, vol. 48, no. 1, pp. 1-17, 2000.

[9] J. R. Vinson, "Sandwich structures," Applied Mechanics Reviews, vol. 54, no. 3, pp. 201-214, 2001.
[10] L. Demasi, "2D, quasi 3D and 3D exact solutions for bending of thick and thin sandwich plates," Journal of Sandwich Structures and Materials, vol. 10, no. 4, pp. 271-310, 2008.

[11] H. Murakami, "Laminated composite plate theory with improved in-plane responses," Journal of Applied Mechanics, vol. 53, no. 3, pp. 661-666, 1986.

[12] E. Carrera, "Historical review of Zig-Zag theories for multilayered plates and shells," Applied Mechanics Reviews, vol. 56, no. 3, pp. 287-308, 2003.

[13] E. Carrera, "Developments, ideas, and evaluations based upon Reissner's Mixed Variational Theorem in the modeling of multilayered plates and shells," Applied Mechanics Reviews, vol. 54, no. 4, pp. 301-328, 2001.

[14] E. Carrera, "On the use of the Murakami's zig-zag function in the modeling of layered plates and shells," Computers and Structures, vol. 82, no. 7-8, pp. 541-554, 2004.

[15] L. Demasi, " $\infty^{3}$ Hierarchy plate theories for thick and thin composite plates: the generalized unified formulation," Composite Structures, vol. 84, no. 3, pp. 256-270, 2008.

[16] S. Brischetto, E. Carrera, and L. Demasi, "Improved bending analysis of sandwich plates using a zig-zag function," Composite Structures, vol. 89, no. 3, pp. 408-415, 2009.

[17] H. Hu, S. Belouettar, E. M. Daya, and M. Potier-Ferry, "Evaluation of kinematic formulations for viscoelastically damped sandwich beam modeling," Journal of Sandwich Structures and Materials, vol. 8, no. 6, pp. 477-495, 2006.

[18] Y. Frostig, "Bending of curved sandwich panels with a transversely flexible core-closed-form high-order theory," Journal of Sandwich Structures and Materials, vol. 1, no. 1, pp. 4-41, 1999.

[19] Y. Frostig and O. Rabinovich, "Behavior of unidirectional sandwich panels with a multi-skin construction or a multilayered core layout-higher-order approach," Journal of Sandwich Structures and Materials, vol. 2, pp. 181-213, 2000.

[20] D. Chapelle and K. J. Bathe, The Finite Element Analysis of Shells_Fundamentals, Springer, Berlin, Germany, 2003.

[21] W. Flügge, Stresses in Shells, Springer, Berlin, Germany, 1960.

[22] A. Scordelis and K. S. Lo, "Computer analysis in cylindrical shells," Journal of American Concrete Institute, vol. 61, pp. 561593, 1964.

[23] J. N. Reddy, Mechanics of Laminated Composite Plates and Shells: Theory and Analysis, CRC Press, Boca Raton, Fla, USA, 2nd edition, 2003.

[24] E. Carrera, "Theories and finite elements for multilayered plates and shells: a unified compact formulation with numerical assessment and benchmarking," Archives of Computational Methods in Engineering, vol. 10, no. 3, pp. 215-296, 2003.

[25] J. Pitkäranta, "The problem of membrane locking in finite element analysis of cylindrical shells," Numerische Mathematik, vol. 61, no. 4, pp. 523-542, 1992.

[26] C. Chinosi, L. Della Croce, and T. Scapolla, "Numerical results on the locking for cylindrical shells," Computer Assisted Mechanics and Engineering Sciences, vol. 5, no. 1, pp. 31-44, 1998.

[27] O. C. Zienkiewicz, R. L. Taylor, and J. M. Too, "Reduced integration technique in general analysis of plates and shells," International Journal for Numerical Methods in Engineering, vol. 3, no. 2, pp. 275-290, 1971.

[28] K. J. Bathe and E. N. Dvorkin, "A formulation of general shell elements-the use of mixed interpolation of tensorial components," International Journal for Numerical Methods in Engineering, vol. 22, no. 3, pp. 697-722, 1986. 
[29] D. N. Arnold and F. Brezzi, "Locking-free finite element methods for shells," Mathematics of Computation, vol. 66, no. 217, pp. 1-14, 1997.

[30] H. Hou-Cheng, "Membrane locking and assumed strain shell elements," Computers and Structures, vol. 27, no. 5, pp. 671-677, 1987.

[31] E. J. Kansa, "Multiquadrics—a scattered data approximation scheme with applications to computational fluid-dynamics. I. Surface approximations and partial derivative estimates," Computers \& Mathematics with Applications, vol. 19, no. 8-9, pp. 127-145, 1990.

[32] Y. C. Hon, M. W. Lu, W. M. Xue, and Y. M. Zhu, "Multiquadric method for the numerical solution of a biphasic mixture model," Applied Mathematics and Computation, vol. 88, no. 2-3, pp. 153175, 1997.

[33] Y. C. Hon, K. F. Cheung, X. Z. Mao, and E. J. Kansa, "Multiquadric solution for shallow water equations," Journal of Hydraulic Engineering, vol. 125, no. 5, pp. 524-533, 1999.

[34] J. G. Wang, G. R. Liu, and P. Lin, "Numerical analysis of Biot's consolidation process by radial point interpolation method," International Journal of Solids and Structures, vol. 39, no. 6, pp. 1557-1573, 2002.

[35] G. R. Liu and Y. T. Gu, "A local radial point interpolation method (LRPIM) for free vibration analyses of 2-D solids," Journal of Sound and Vibration, vol. 246, no. 1, pp. 29-46, 2001.

[36] J. G. Wang and G. R. Liu, "A point interpolation meshless method based on radial basis functions," International Journal for Numerical Methods in Engineering, vol. 54, no. 11, pp. 16231648, 2002.

[37] J. G. Wang and G. R. Liu, "On the optimal shape parameters of radial basis functions used for 2-D meshless methods," Computer Methods in Applied Mechanics and Engineering, vol. 191, no. 23-24, pp. 2611-2630, 2002.

[38] X. L. Chen, G. R. Liu, and S. P. Lim, "An element free Galerkin method for the free vibration analysis of composite laminates of complicated shape," Composite Structures, vol. 59, no. 2, pp. 279-289, 2003.

[39] K. Y. Dai, G. R. Liu, K. M. Lim, and X. L. Chen, "A mesh-free method for static and free vibration analysis of shear deformable laminated composite plates," Journal of Sound and Vibration, vol. 269, no. 3-5, pp. 633-652, 2004.

[40] G. R. Liu and X. L. Chen, "Buckling of symmetrically laminated composite plates using the element-free galerkin method," International Journal of Structural Stability and Dynamics, vol. 2, pp. 281-294, 2002.

[41] K. M. Liew, X. L. Chen, and J. N. Reddy, "Mesh-free radial basis function method for buckling analysis of non-uniformly loaded arbitrarily shaped shear deformable plates," Computer Methods in Applied Mechanics and Engineering, vol. 193, no. 3-5, pp. 205224, 2004.

[42] Y. Q. Huang and Q. S. Li, "Bending and buckling analysis of antisymmetric laminates using the moving least square differential quadrature method," Computer Methods in Applied Mechanics and Engineering, vol. 193, no. 33-35, pp. 3471-3492, 2004.

[43] L. Liu, G. R. Liu, and V. B. C. Tan, "Element free method for static and free vibration analysis of spatial thin shell structures," Computer Methods in Applied Mechanics and Engineering, vol. 191, no. 51-52, pp. 5923-5942, 2002.

[44] S. Xiang, K. M. Wang, Y. T. Ai, Y. D. Sha, and H. Shi, "Analysis of isotropic, sandwich and laminated plates by a meshless method and various shear deformation theories," Composite Structures, vol. 91, no. 1, pp. 31-37, 2009.

[45] S. Xiang, H. Shi, K. M. Wang, Y. T. Ai, and Y. D. Sha, “Thin plate spline radial basis functions for vibration analysis of clamped laminated composite plates," European Journal of Mechanics A, vol. 29, no. 5, pp. 844-850, 2010.

[46] C. M. C. Roque, A. J. M. Ferreira, and R. M. N. Jorge, "Free vibration analysis of composite and sandwich plates by a trigonometric layerwise deformation theory and radial basis functions," Journal of Sandwich Structures and Materials, vol. 8, no. 6, pp. 497-515, 2006.

[47] A. J. M. Ferreira, "A formulation of the multiquadric radial basis function method for the analysis of laminated composite plates," Composite Structures, vol. 59, no. 3, pp. 385-392, 2003.

[48] A. J. M. Ferreira, "Thick composite beam analysis using a global meshless approximation based on radial basis functions," Mechanics of Advanced Materials and Structures, vol. 10, no. 3, pp. 271-284, 2003.

[49] A. J. M. Ferreira, C. M. C. Roque, and P. A. L. S. Martins, "Analysis of composite plates using higher-order shear deformation theory and a finite point formulation based on the multiquadric radial basis function method," Composites Part B, vol. 34, no. 7, pp. 627-636, 2003.

[50] A. J. M. Ferreira, C. M. C. Roque, and R. M. N. Jorge, "Modelling cross-ply laminated elastic shells by a higher-order theory and multiquadrics," Computers and Structures, vol. 84, no. 19-20, pp. 1288-1299, 2006.

[51] E. Carrera, " $\mathrm{C}^{0}$ reissner-mindlin multilayered plate elements including zig-zag and interlaminar stress continuity," International Journal for Numerical Methods in Engineering, vol. 39, no. 11, pp. 1797-1820, 1996.

[52] E. Carrera and B. Kröplin, "Zigzag and interlaminar equilibria effects in large-deflection and postbuckling analysis of multilayered plates," Mechanics of Composite Materials and Structures, vol. 4, no. 1, pp. 69-94, 1997.

[53] E. Carrera, "Evaluation of layerwise mixed theories for laminated plates analysis," AIAA Journal, vol. 36, no. 5, pp. 830-839, 1998.

[54] R. L. Hardy, "Multiquadric equations of topography and other irregular surfaces," Geophysical Research, vol. 176, pp. 1905-1915, 1971.

[55] A. J. M. Ferreira and G. E. Fasshauer, "Computation of natural frequencies of shear deformable beams and plates by an RBF-pseudospectral method," Computer Methods in Applied Mechanics and Engineering, vol. 196, no. 1-3, pp. 134-146, 2006.

[56] J. N. Reddy and C. F. Liu, "A higher-order shear deformation theory of laminated elastic shells," International Journal of Engineering Science, vol. 23, no. 3, pp. 319-330, 1985. 


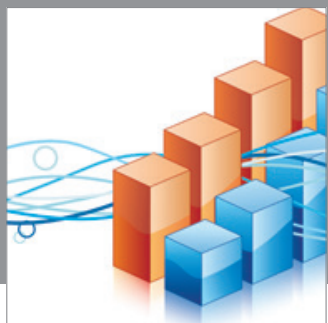

Advances in

Operations Research

mansans

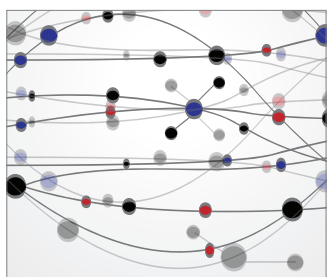

The Scientific World Journal
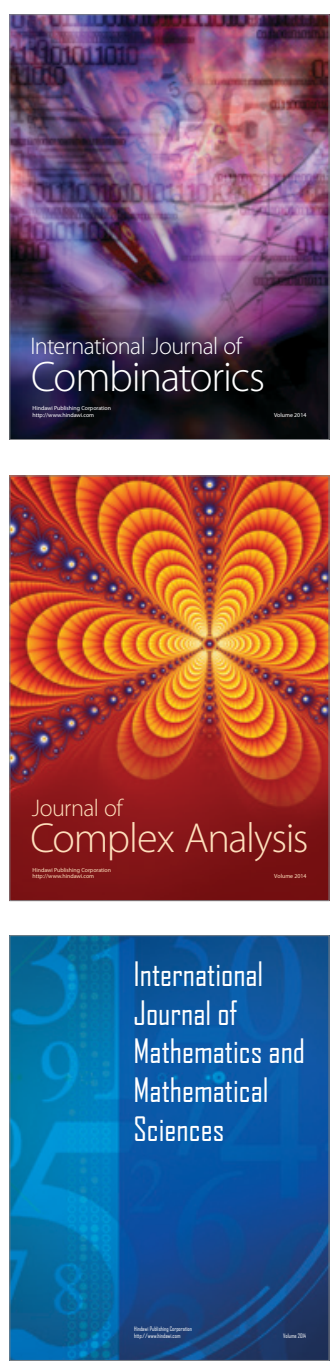
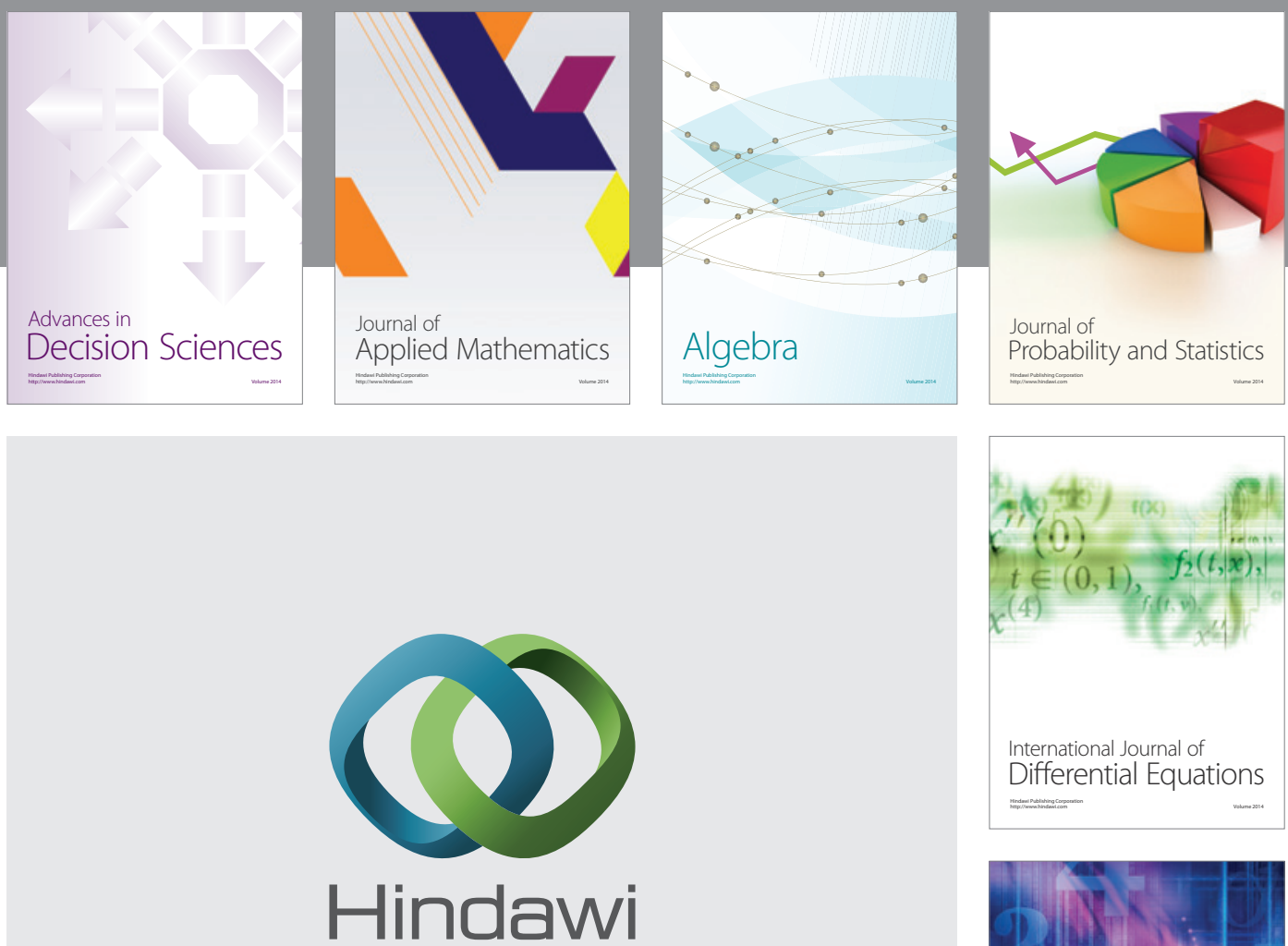

Submit your manuscripts at http://www.hindawi.com
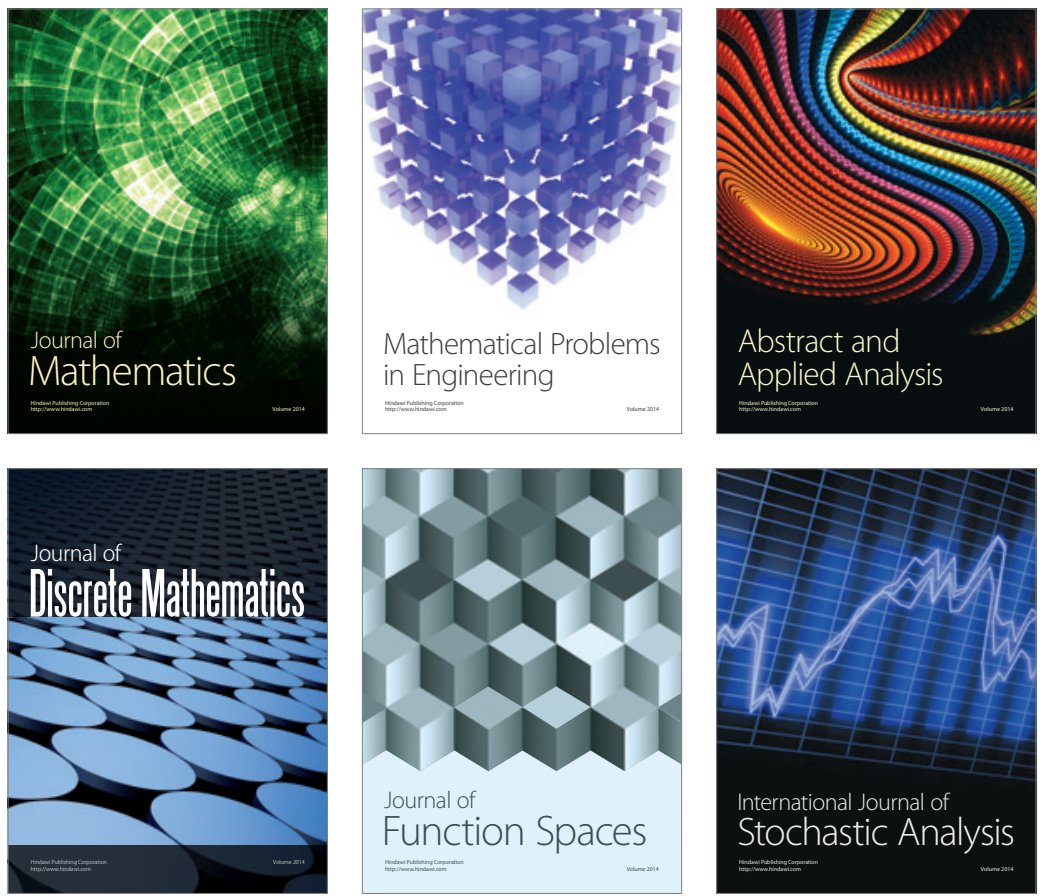

Journal of

Function Spaces

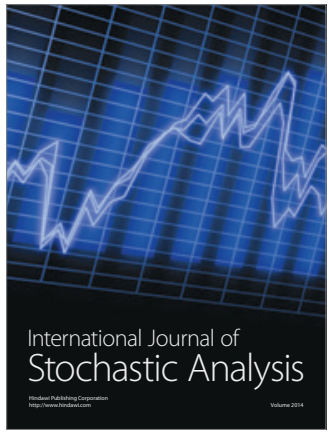

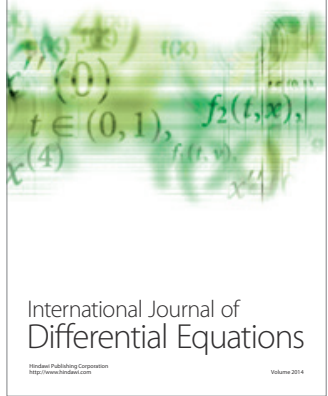
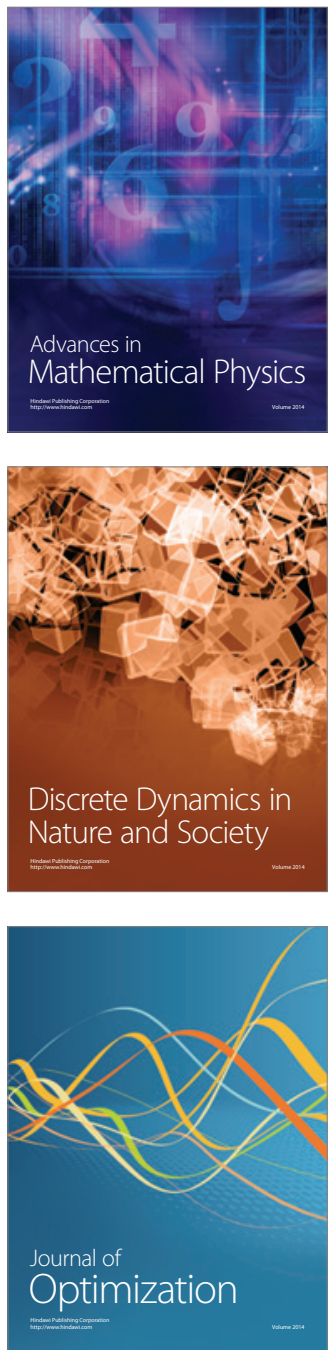